\title{
BIBLIOTECA
}

DEL

\section{Circolo Matematico di Palermo}

\section{PUBBLICAZIONI INVIATE DAGLI AUTORI (*)}

M. L. Albeggiani (Palerno). Sviluppo d'uu determinante ad elementi polinomt, due note. Giornale di Battaglini, vol. X, XIII.

Dimostrazione d'una formoia d'analisi di F. Lucas, ibid., vol. XIII.

Geometria dello spazio in coordinate tetraedriche secondo $\mathrm{i}$ concetti delle Volles. über Geom. di A. Clebsch, Palermo, 1877.

Intorno ai concetti ed ai metodi fondan.entali della Geometria analitica. Prolusione, Palermo, 1878.

G. Battaglini (Roms). Soprd slcune propriet d delle superficie di secondo grado, 1857 .

Sulla dipendenza scambievole delle figure. ' $R$. Acc. del'e scienze fisiche - matematiche di Napoli.

Sulld partizione dei numeri, ibud.

Soprd alcune proprietd deite linee di $2^{\circ}$ grado, $i b t d . ~ 1862$.

Sulle superficie di $2^{\circ}$ grado, tbid. 1862.

Nota soprd alcune quistioni di Geometriı, tbid. 1862.

Notd di geometria, ibid. 1862 .

Sulle forme geometriche, $t b t d .1862$.

Nota sui determinanti, ibtd. 1862.

Sopra und questione di massimi e minimı, $t b ı d$. 1863 .

Sulle serie di curve d'indice qualunque, thad. 1863 .

Sulla dipendenzs equidnarmonica, ibid. 1863 .

Sulla dipendenza di primo ordıne, 2 bud. 1863.

Sulle involuzioni dei diversi ordini, ibil. 1863.

Sulla dipendenza duplo-anarmonica, ibid, $\{86\}$.

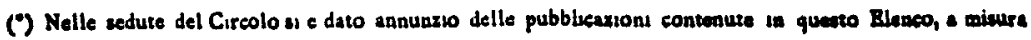
the was stale stearate. 
G. Battaglini (Roma). Teorid elementare delle forme geometriche. Giorn. di Battaglini, t. 1, 1863 .

Sulle divisioni omografiche immaginarie. R. Acc. di Napoli, 1864.

Sulle forme binarie di $1^{\circ}$ e di $2^{\circ}$ grado, ibid. 1864.

Sulle forme binarie di $3^{\circ}$ grado, ibid. 1864.

Sulle forme binarie cubiche, ibid. 1864 .

Sulle forme binarie di $4^{\circ}$ grado, ibid. 1864 .

Sulle forme binarie biquadratiche, ihit, 1864 .

Sulle forme binarie biquadratiche in involuzione, ibid. 1864 .

Sulle forme binarie miste di $3^{\circ}$ e $4^{\circ}$ grado, ibid. 1864 .

Sopra una curva di $3^{\star}$ classe e di $4^{\circ}$ ordine, ibid. 1865 .

Sulle forme geometriche di $2^{2}$ specie, $2 b \pm d$. 1865 .

Sulle forme geometriche di $2^{2}$ specie, ibid. 1865 . Nota $2^{*}$.

Sulle involuzioni dei diversi ordini nei sistemi di $2^{2}$ specie, thid. 1865 .

Sulle forme binarse dei primi quattro gradi appartencnti ad una forma ternaria quadratica, ibid. 1865 .

Intorno ai sistemi di rette di primo ordine, ibid. 1866.

Intorno di sistem di rette di secondo grado, ibid. 1866.

Sulle forme binarie dei primi quattro gradi, appartenenti ad und forma ternaris quadratica. Nota seconds, ibid. 1866.

$$
\text { - - - Nota terza, ibid. } 1866 .
$$

Osservazione intisno ad una formola relativa all' elettrometro bifiliare, ihid, 1866 .

Intorno ai momentı geometrici di $I^{\circ}$ grddo. Notd prima, ibid. 1866.

Sulla geometris imnaginaris di Lobatschewsky, tbid. 1867 .

Sulle forme binarie di grado qualunque, tbud. 1867.

Sulle forme ternarie quadratiche. Memoria prima, $2 b u d .1867$.

$$
\text { - -- Memoria seconda, ibid. } 1867 \text {. }
$$

Intorno ai sistemi di rette di grado qualunque, ibtd. 1868.

Sulle forme ternarie di grado qualunque, เbid. 1868.

Sulla composizione delle forze, ibid. 1869 .

Sulls teorica dei momenti, tbid. 1869.

Sulle serie dı sistemı d forze, ibid. 1869.

Sulle dinami in involuzione, $2 b t d .1869$.

Sul movimento geometrico infinitesimo d'un sistema rigido, 3bid. 1870.

Sul movımento geometrico finito d'un sistema rigido, ibid. 1870 .

Sulla teorica dei momenti d'inerzia, ibid. 1871 .

Sul movimento di un sistema di forma invariabile, tbid. 1871 .

Nota intorno alla Conica rispetto alla quale due Coniche date sono polari reciproche tra di loro. ' $R$. Accademia dei Lincei, 1872 .

Nota intorno alla Quadrica rispetto alla quale due Quadriche date sono polari recipreche tra di loro, ibid. 3872 .

Nota sul rapporto anarmonico sezionale e tangenziale delle Coniche, ibid..1873. 
G. Battaglini (Roma). Nota sui circoli nella Geometria non-euclidea. $R$ Accade. mia dei lincei, 1873 .

Sulla geometrid proiettua. R. Acc. de Napoli, 1873 .

- Memoria seconda, ibrd. 1874 .

Nota sul rapporto anarmonico sezionale e tangenziale delle quadriche. $R$. Ace. dei Lincei, 1874 .

Nota intorno ad una superficie di $8^{\circ}$ ordine, ibid, 1875.

Sulla quintica binaria, ibid. 1875 .

Sulla Geometria proiettiva. Memoria terza. R. Acc. di Napoli, 1875.

Sull'Affinith circolare non-euclidea, ibid. 1876.

Sul movimento per una linea di $2^{\circ}$ ordine, ibid. 1877 .

Sui complessi di secondo grado. $R$. Acc. dei Lincei, 1878 .

Sulle cubiche ternarie suzigetiche, 1879 . Estratto dal volume Collectanea Mathematzca in memoriam $D$. Chelini, Milano $188 \mathrm{r}$.

Sui connessi ternart $\mathrm{d}_{1} 2^{\circ}$ ordine e di $2^{\mathrm{a}}$ classe in involuzione semplice. R. Acc. di Napoli, 1879.

Sull' equazione differenziale ellittica. R. Acc. dei Lincei, 1879.

Sui connessi ternart di $\mathrm{t}^{\circ}$ ordine e di $\mathrm{I}^{\star}$ clusse. R. Acc. di Napoli, 1880.

Sulle forme ternarie bilineari. $R$. Acc. dei Lincei, 1880.

Sulle forme quaternarie bilineari, ibid. $188 \mathrm{r}$.

Sopea una quistione di Geometria proiettiva. $R$. Istituto d' incoraggiamento alle scienze naturali economiche e tecnologiche in Napoli, 1882.

Collezione dei primi diciotto volumi del « Giornale di Matematiche ad uso degli studenti delle Universitd italiane ", 1863-80, Napoli, B. Pellerano Editore.

27. Bortini (Pavia). Libro quinto d'Euclide. Roma, 1874.

Sui complessi di $2^{\circ}$ grado. Giom. di Batt., vol. XVII.

Sui sistemi lineari. $R$. Istituto Lombardo, 12 gennajo 1882 .

Costruzioni geometriche della trasformazione univoca di $3^{\circ}$ ordine, ibid. 23 febbrajo 1882 .

Contribuzione alla teoria delle 27 rette e dei 45 piani tritangenti di una superficie di $3^{\circ}$ ordine. Annals di Matematica, serie $2^{*}$, t. XII.

I. Bianchi (Pisa). Sulle superficie applicabili. Pisa, 1878 .

Ricerche sulle superficie a curvatura costante. Pisa, 1879.

Ueber die Normalformen dritter und funfter Stufe des elliptischen Integrals erster Gattung. Math. Annalen, Band XVII.

Sui sistemi tripli ciclici dı superficie ortogonali. Giornale di Batt. vol. XXII. Sopra una proprietà caratteristica delle superficie ad area minima, ibid. XXII. Sopra i sistem tripli ortogonali di Weingarten. R. Acc. dei Lincei, Rendiconti, vol I, Is febbrajo i885.

- nota seconda, ibtd. is marzo 1885 .

Sopra una classe di sistemi tripli di superficie ortogonali, che contengono un sistema di elicoidi aventi a comune l'asse ed il passo. Annall di acatematica, serie $2^{*}$, t. XIII, 1885 . 
F. do Boor (Leiden). Extension du thicorème de Rolle. Archives Nlorlandafses, t. XIX, novembre 1883 .

F. Caldarera (Palermo). Sulla determin tzione delle latitudini ed azimuti degli oggetti terrestri, e l'equazione di un orologio che va a tempo siderio. Atti Aec. Giosnia di Catania, 1854.

Sulla Trigonometria. Giorn. della $R$. Specola di Palerno, 1856.

Sopr.3 und proposizione contenum nella teoria delle funzioni ellittiche di Legendre. Giom. dellu societd di scienze natural ed economiche in $\boldsymbol{P}_{a-}$ lermo, 1865 .

Sul teorema di Legendre per la risoluzione dei triangoli sferiti pochissimo curvi, ibid. 1865 .

Dei determinanti a matrice magica, ibid. 1866.

Sulla formola comunemente adoperats pel calcolo degli archi di meridiano, Palermo 1866.

Su talune proprietà dei determinanti, in ispecie di quelli \& matrici composte con le serie dei numeri figurati. Giorn. di Batt., vol. IX.

Sullo sviluppo delle funzioni a varidbili piccolissime, ibid. XII.

Lezioni di Meccanica razionale per l' anno sc. 1879-80. Parte litografata : Cinematici-Studio delle forze.

Introduzione allo studio della Geometriı superiore, vol. I, Palermo, I880.

A. Capalli (Palermo). Sopri l'isomorfismo dei gruppi di sostttuzioni. Giorn, di Batt., vol. XVI.

Sopra un punto deli، teoria delle forme binane, tbid. XVI.

Sopes la corrispondenza $(2,2)$ ossia la forma $f\left(x^{2} y^{2}\right)$ ed i suoi invarianti e covarianti relativi a due trasformazioni linean indipendenti delle variabili, ibid. XVII.

Sopra le forme algebriche ternarie a piì serie di variabili, ibtd. XVIII.

Sopra gli invarianti delle forme algebriche binane. Giorn. di Scienze Naturali ed Economicbe, vol. XV, 1880. Palermo.

Fondamenti $\mathrm{d}_{1}$ una teoria generale delle forme algebriche. $R$. Ace. dei Linces. Memorie, vol. XII, S marzo I882.

Sul numero dei covarianti di grado dato per forme di qualsivoglia specie. Grorn. di Batt., vol. XX.

Estensione della forinola pel numero dei covarianti al caso delle trasformazioni lineari indipendenti. R. Acc. dei Lincei, Memorie, vol. XV, 4 marzo 1883.

Alcune formole numerithe in relazione alla teorid delle operazioni di polare. Giorn di Batt., vol. XXI.

Sopra la composizıone dei gruppi di sostituzione. R. Ace. dei Lincei, Memorie, vol. XIX, 2 marzo 1884 .

B. Caporali (Napoli). Sulla superficie del quinto ordine dotata d'una curva doppia del quinto ordine. 6 luglio 1875 . Ann. di Matematica, serie $2^{*}$, t. VII.

Teoremi sulle curve del terzo ordine-Teoremi sui fascl di curve del terzo ordine. R. Acc. dei Lincei, 17 giugno 1877 . Transunti. 
E. Caporall (Napoli). Sopra i piani ed i punti singolari della superficie di Ku mm er, R. Acc. dei Lincei, 2 giugno 1878, Memorie della Classe di scienze fis. mat. e natur. vol. II.

Sui complessi e sulle congruenze di $2^{\circ}$ grado, ihid., ibid.

Sopra i sistemi hnearı triplamente infiniti di curve algebriche piane, luglio 1879.

Estratto dal volume Collectanea Mathematica in memoriam Chelini. Milano, $188 \mathrm{I}$.

Sulle trasform.zioni univoche piane involutorie. $R$. Acc. delle Scienze Fis.e

Matem. di Napoli, settembre 1879 . Rendiconti.

Sopra alcuni sistemi di rette, ibul., II ottobre 1879 . Rendiconti.

Sull' essedro completo, tbid., 5 febbrajo $\mathrm{i} 38 \mathrm{r}$. Rendiconti.

Teoremi sulle superficie del $3^{\circ}$ ordine, ibial., $7 \mathrm{maggı} 188 \mathrm{I}$. Rendiconti.

Sulle t.ıngenti condotte ad una curva algebrica piana da un suo punto inultiplo, ibid., II giugno $188 \mathrm{I}$. Remliconti.

Sopr. un.t cert $\lrcorner$ curva del $4^{\circ}$ ordine, thid., 9 dicembre 1882. Rendiconti.

Sul sıstema di due frrme binarie cubıche, ibil, $10 \mathrm{~m} 1570$ 1883. Rendiconti.

Relazione sul concorso pel Premio aceidemico dell'anno 1882 , zbid., dicembre 1883. Rendiconti.

F. Casorati (Pavia). Intorno ad alcuni punti dell $\_$teori $\lrcorner$dei minimi quadedti. Ro$m a, 1858$.

Teorica delle funzıoni di variabili complesse. Volume primo. Pavis, 1868.

Sopra la determinazıone delle alterazıom nei valori di somme e prodotti infiniti dovute dd alterazionı nell'ordıne dı addizione o moltuplicazione dei terminı o fattori. Rendiconti R. Ist. Lomb., serie $2^{\mathrm{a}}$. vol. I, 1868.

Un teorema fondamentale nella teorica delle discontinuit delle funzion, $1 b$ bd.

Le relazioni fondamentah tra i moduli di periodicıta degli integrali abeliani di ${ }^{2}$ specie. Ann. di Mat., serie 2". t. III, 1869.

Alcune formole fond.ımentali per lo studio delle equazioni algebrico-differenziali di $1^{\circ}$ ordine e $2^{\circ}$ grado tra due variabıli ad integrale generale algebrica. $R$. Istit. Lomb., 1874 .

Sulla regola seguita da Bessel e dal sig. Generale Baeyer durante la misurd del gr.do nella Prussi.t orientule, etc. Alli lella R. Ace. dei Lincoi, serie $2^{\mathrm{n}}$, t. II, 1875 .

Sui determinanti di funzione. Milano, 1875 .

Le proprietd cardinals degli strumenti ottici anche imperfettumente centrati.

In memoria di Jacopo Steiner. Traduzione dal tedesco. Ann. di Mat. serte $2^{\text {a }}$, t. VII, 1875 .

Nuova teoriา delle scluzioni singoldri delle equazion dafferenzisli di $x^{\circ}$ ordine e $2^{\Delta}$ grado tra due variabli. Atti $R$. Ace. dei Lincei, serie $2^{\text {a }}$ t. III. 1876 .

Sulle coordinate dei punti e delle rette nel piano, dei pu ti e dei piani nello spazio. R. Ist. Lomb., 1877 .

Sulle condzioni alle quali deve soddisfare und prinitiva affinche il grado 
della corrispondente equazione differenzinle, risperto alle variabili, riesca minore del normale, R. Ist. Lomb., 1877.

F. Casorati (Pavia). Sulla integrazione delle equazioni algebrico-differenzidi di $x^{\circ}$ ordine $c$ di $1^{\circ}$ grdúo per mezzo di funzioni lineari, ibid., $187^{8}$.

Nuova e migliore forma delle equ.uzioni degli asintoti di una linea piana algebrica, ibid., 1879 .

Nota concernente la teoris delle soluzioni singolari delle equazioni algebricodifferenziali di $1^{\circ}$ ordine e di $2^{\circ}$ grado. $R$. Acc. dei Lincei, 1879.

Il calcolo delle differenze finite interpretato ed accresciuto di nuovi teoremi a sussidio principalmente delle odierne ricerche basute sulla variabilita complessa, ibid., 1880 .

Sur la distınction des intégrales des équations diffrentielles linédires en sousgroupes. Comples Rendus, 24, 31 junvier 1881.

Una formol.s fond.ument.ale concernente $i$ discrunin.unti delle equ.zioni differenzili e delle loro primitive complete. D.l vnlume Collectanea Mathematica in mert. Chelini, Milano, 1881.

Nota di mitematica pura. R. Acc. dei Lineei, 1881 .

Generalizzazione di alcuni teoremi dei signori Hermite, Brioschi e Mittag-Leffler, sulle equazioni differenziali lineari del $2^{n}$ ordine. Ann di Mat. serie $2^{2}$, t. X 188 .

Sur un érit très-récent de M. Stickelberger. Pavia, 188ı.

Sulle equazioni differenzi h lineiri. $R$. Acc. dei Lincei, 1882.

Aggunte a recentu lavori dei signori Weicrstrass e Mittag-Leffler sulle funzioni di uns varubile complessa. Ann. di Mat. serie $2^{\circ}$ t. X. 1882 .

La periodicrti nultipla nelle funzıoni di un $s$ sola varisbile. $R$. Ist. Lomb. 1883 . Sopra alcuni discrimin.unti, ibzd., 1885 .

F. Chiszoni (Rom). Sulle superficie e sulle linee che si ottengono come luogo o come inviluppo delle rette conglungenti i punti corrispondenti di due curve omogrufiche piane. R. Ace. dei Lincei, Memorie, vol. III, 5 gennujo 1879 .

Soprs le involuzion nel piano, ibid. XIX, 3 giugno 1883.

L. Cremona (Rom1). Sulle tr.sformazioni rizionali nello spazio. Annali di ACalematica, serie $2^{*}, t . V$.

R. De Paolis (PIsu). L 1 trasformazione pun 1 doppia di terzo ordine primo genere e l.t sua applicazione alle curve del quarto ordine. R. Acc. dei Linces, Kemorie, vol. II, 5 maggio 1878 .

Ricerche sulle superficie del $3^{\circ}$ ordine, due Memorie, ibid. X, $188 \mathrm{I}$.

Sulla espressione di uns forms binaria di grado $n$ con una somma di potenze $n^{e}$, tbul. XII, 5 marro 1882 .

E. Fergola (Nupoli). Sopra talune proprietd delle soluzioni intere e positive dell'equazione $x_{1}+2 x_{2}+\ldots+n \alpha_{n}=n$. Rendiconti della $R$. Acc. delle Scienze Fis. e MCut. di Nippoli, ottobre 1863 . 
E. Fergola (Napoli). Sopra una proposizione elementare di Calcolo !integrale. Rendiconti della $R$. Ace, delle Scienze Fis. A Mcut. di Napoli, ottobre 1864 .

Sulla posizione dell'asse di rotazione della terrs rispetto all'asse di figura. Annali di MCatematica, serie $2^{4}$, t. VI, 1874 .

Dimensioni della terra e ricerca della posizione del suo asse di figura rispetto d quello di rotazione. Rendiconti della $R$. Acc. di Napoli, dicembre 1875 .

$\mathrm{Di}$ dlcune equazioni relative alla tenria delle funzioni ellittiche e teoremi di Geometria che vi si connettono. ACamorie della Societd italiana delle Srienze, vol. IV, 20 maggio 1882.

Sulla lititudine del R. Osservatorio di Capodimonte. Atti della R. Acc. delle Scienze di Napoli, vol. I, serie $2^{2}$, $\mathrm{I}^{0}$ dicembre 1883 .

G. Frattini (Rom1). Intorno ad un teorema di Lagr ınge. R. Act. dei Lincei. Rentdiconti, $1^{\circ}$ febbrijn 1885 .

Un tenrem! relativo al gruppo della trasform izione modulare di grado $p$. Due note, ibid. I e is febbrajo 1885 .

Intorno dlla generazione dei gruppi di operazione, ibtd. 12 aprile 1885.

P. Gambera (P.lermo). Dells velocits e delld energha delle molecole dei fluidi aeriformi. Palermo, 1884 .

M. Gobbia (P.slermo). Sulla stabilta virtudle deli'equilibno d'un punto materiale isolato. Giorn. di Bitt. XVI.

Le travature reticolari a membri soviabbondanti. Alti del Collegio degli Ingegneri ed Arcl. di Palermo, I88I.

Determin zzione grafica degli sforzi interni nelle tr.svature reticolari con aste sovrabbond.unti. $R$. Ace. dei Lincet, Memorie, vol. IX, 6 marzo I88I.

Singli sforzi interni dei sistemi articolati, tbid. XIII, 2 aprile 1882.

P. Gordan (Erlangen). Ueber endliche Gruppen linearer Transformationen einer Ver.underlichen. Mathemalisclse Annalen, Band XII, 1877 .

Binure Formen mit verschwindenden Covarianten, ihud. XII, 1877.

Ueber das volle Formensystem der ternaren biquadratischen Form $f=x_{1}^{3} x_{2}+x_{2}^{3} x_{3}+x_{3}^{3} x_{1}$, ihd. XVII, 1880 .

Gordan-Clebsch. Ueber cubische ternure Formen, thid. VI, 1873 .

G. B. Guccia (Palermo). Sur une classe de surf.ces reprúsentables point par point sur un plin. Assocution française pour l'avancement des Sciences. Congris de Reints, 1880.

G. Halphen (Paris). Sur certuines perspectives gauches des courbes planes algébriques. Comptes Rendus, is mars 1875 .

Sur les invariants des equations différentielles linéaures du quatriense ordre. Acta Mathemattca, III.

Etude sur les points singuliers des courbes algébriques planes. Appendice au Traite des courbes planes de G. Salmon. Paris, Gauthier-Villars, 1883.

T. A. Hirst (London). Ueber conjugirte Diameter in dretaxigen Ellipsoid. Inaugural-Dissertation. Murburg, 1852 . 
T. A. Hirst (London). On two new methods of defining Curves of the second order, ingether with new properties of the sume deducible therefrom. By Professor Stein er. Translated by Dr. T. A. Hirst. Cambridge and Dublin Mathematical Journal, nov. 1853 .

On equally attracting bodies. From the Philosophical Magazina for sept, and oct. 1858 .

M, Poinsot on the Percussion of Bodies, ihid.

Note sur les corps qui exercent des attractions égules sur un point matériel. Comptes Rendus, XLVII, 9 dout, 1858 .

Sur la courbure d'une série de surfaces et de lignes. Annali di Matematica, t. II, 18 s.

On derived Surfaces. Quarterly Journal, july 1859.

On ripples, and their relation to the velocities of currents. Plulosophicul Magazine for j.unuary and march $186 \mathrm{r}$.

On the Volumes of Pedal Surfuces. Proceelings of the Ruyal Society, 1862.

On the Volumes of Pedal Surfices. Philosopitscal Transactions of the Royal Society, vol. 153, 1862 .

Sur les volumes des surfaces podaires. Crelle's Jourmal, Band. 62.

Sur les volumes des surfices podaires. Annali di ACatematica, t. V, 1863.

On normals to conics, a new treatment of the subject. By Prof. Cremond. Communicated by T. A. Hirst. The Oxford, Cambridge, and Dublun MCessenger of MCathematics, No. 10, 1865 .

Sull' inversıone quadrica delle curve pi..ne. Annali di Matematica, t, VII, 1865 .

Discorso pronunzidto dall' Autore in oscisione del conferimento della medaglia Cople y.3. M. Ch . s le s. Proceding's of the Royal Society No. 79, 1865.

Sur la transformation quadrique. Nouvelles Annules de Muthemuatiques, $2^{\circ}$ série, t. VI, 1866.

On the degenerate Forms of Conics. Proceedings of the London Mathem. Society, vol. II, 1869 .

On the Correlation of two Planes. Due memorie, ibid. vol. V, 1874; vol. VIII, 1877 .

On the Correlution of two Planes. Due memorie. Annali di Matematica, serse $2^{2}$, t. VI, VIII.

On Correlation in Space. Proceed. London Mathens. Soczety, vol. VI, 1874 . Geometric.1 Contributions to the "Educational Times". London, 1875 .

Sur la Corritation de deux plans. R. Acc. dei Lincei, Transunti, Serie $3^{2}$, vol. I, 4 marzo 1877 .

Extracted from the Sixth General Report of the Association for the Impro. vement of Geometricul Teaching, january 1878 .

Note on the Complexes generated by two Correlative Planes. Proceed. of the London Mathem. Society, vol. X, 1879.

On the Compleves generated by two Correlative Plsnes. Collecluneu Mathemubica in memortum Chelini, Mediolani, 188 . 
T. A. Hirst (London). On qu.tdric Teunsformution. Quarterly Journal, N. 68, 1881. « The Biograph and Review », september 1881 .

On Cremonian Congruences. Proceal. of the London Mathern. Sociely, volume XIV, 1883.

2. de Jonquieres (Paris). De la représentation des nombres par des formes qua. dratiques bin.ires. Applicution d l'analyse indeterninie. Assoc, franc. pour l'avanc. des Sciences, Congres de Paris, 1878 .

Note sur un point de 12 théorie des fractions continues périodiques. Comptes Rendus de l'Acad. des Scientes, 26 février 1883.

Sur la composition des périodes des fractions continues périodiques, ibid. 12 mars 1883 .

Addition aux Communications précédentes sur les fractions continues périodiques, ibid. 26 mars 1883 .

Loi des púriodes. Trois Communications, ibid. 9, 16, 23 avril 1883.

Sur les fractions continues périodiques dont les numerateurs différent de l'unite. ibtd. 30 avril 18.33 .

Étude des identités qui se présentent entre les reduites appartenant, respectivement, aux deux modes de fractions continues périodiques-Cinq Communications, ibid. $7,14,21,28$ mai, 4 juin 1883.

Sur le dernier thiorime de Ferm at. atti dell Accademia Pontificta det Nuvvt Lincei, t. 37,20 gennuro 1884 .

Conmentuire arithmitique sur une formule de Gauss. Comptes Rendus, 2 juin 1884.

Sut lit régle de Newton (dimnntré par M. Sylvester), pour trouve: le nombre des racines inuginuirus des équations numériques, ibid. 14 , 21,28 juillet $188_{4}$.

Sur les equations algébriques, ibt. 1 ; 22 septembre 1884.

Mímorte sur les figures isogruphiques et sur un mode uniforme de génesration des courbes à double courbure d'un ordre quelconque du moyen de deur fiscedux correspondints de droites. Giornale di Battaglini, vol. XXIII, 1885 .

It Vice-Ammiraglin de Jonquieres ha inoltre unviato la sua fotografil colla dedica al Circolo.

C. Jordan (Paris). Recherches sur les polyèdres. Journal de Crelle, t. 66, 1866. Recherches sur les polyédres. Second mémoire, ibid. t. 68, 1868.

Mémorre sur la stabiluté de l'équilibre des corps flottants. Annali di SCatematica, 1868.

Commentaire sur Galois. Malbenatische Annalen, t. I, 1869 .

Sur les équations de $\mathrm{I}$ division des fonctions abéliennes, tbil. t. I, 1869.

Thèorìmes sur les équatınns algébriques. Journal de Liouville, $2^{\mathrm{e}}$ série, t. XIV, 1869.

Sur l'iquation aux 27 droites des surfaces du $3^{\text {tme }}$ ordre, ibid. 1869.

Sur les assemblages de lignes. Journal de Crelle, t. 70, 1869. 


\section{8}

C. Jordan (Paris). Sur une équation du 16 tme degré. Journal de Crelle, t. 70, 1869.

Sur une nouvelle combinaison des 27 droites d'une surface du' 3 cme ordre. Comptes Rendus, t. 70, 1870 .

Thioreme sur les fonctions doublement périodiques, ibid. 1870 .

Theorèmes sur les groupes primitifs. Joum. de Liourville, 2e série, t. XVI, 1871 . Sur ld résolution des équations les unes par les autres. Comples Reudus, 1871 . Sur les sommes de $G$ a uss a plusieurs variables, ibid. 187 1.

Sur les oscillations infiniment petites des systèmes matériels, ibid. 1872 . Recherches sur les substitutions. lourn. da Liouville, $2^{\circ}$ série, t. XVII, 1872.

Sur la forme canonique des congruences du second degré et le nombre de leurs solutions, ibud.

Mémoire sur la réduction et la transformation des systèmes quadratiques, tbid. t. XIX, 1874 .

Sur la' limite du degré des groupes primitifs qui contiennent une substitution donnie. Journal de Crelle, t. 79, 1874 .

Sur la stabilité de l'équalibre d'un solide pessnt posé sur un appui courbe. Journal de Liouvblle, ze sćrie, t. I, 1875 .

Mimoire sur les coviriants des formes bin.ires, ihtd. $3^{\text {e }}$ serie, t. II, 1876 .

Mémoire sur les ciractéristiques des fonctions $\Theta$. Journal de $l$ Ecole Polysechnique, 1879 .

Sur les covariants des formes bin tires, deuxième nèmoire. Journ. de Liouville, ze série, t. V, 1879 .

Sur l. réduction des substitutions linéaures. Journ. del'Ecole Polyteclinique, 1880.

Mćmoire sur l'équivalence des formes, ibid. $188 \mathrm{n}$.

Sur $I_{t}$ theorie arithmétıque des formes quadratiques, ibid, 1882.

G. Jung (Milıno). Sopra alcuni tenremi di $\mathrm{G}_{2}$ uss intorno alla teorica della ripartizione del circolo. Assoc. delle conferenze di Matenuttche, 24 giugno 1867.

Jung-Armenante. Sulle tr isformazioni birazionali o univoche (eindeutigen) e sulle curve normale e subnornale del genere p. Giornale di Battaglini, vo. lume VII, 1869 .

Relazione sulle lezioni complementali date nel R. Istituto Teenico superiore di Milano dil prof.' Brinschi, Cremona e $C_{d}$. sordi, ibid.

G. Jung (Milano). Intorno ai momenti d' inerzia di una sezione piand e di diversi modi di rappresenta.li graficamente; in particoldre dell'ellisse centrale, della sua curva pedale e del circolo d' inerzia. Rendiconti dell' Istituto Lombarido, 29 luglio 1875

Rappresent.ı?ıoni grafiche dei momenti resistenti di unı sezione pian ı, ibid. 6 Juglio 1876.

Complemento alla nota precedente, ibid. 8 agosto 1876 .

On a new Construction for the Central Nucleus of a Plane Section. British Association for the Advanc. of Science, 1876 .

Risumi of Restirches upon the Gruplical Representution of the Moments of Resistance of Plane Figures, ibid. 1876. 
G. Jung (Miluno). Thtororeme général sur les fonctions symétriques d'un nombre quelconque de variables. Comptes 'Rendus des seances de $l$ Acad. des Sciences 24 avril 1876 .

Construction de la chainette par points, et division d'un are de cette courbe en $n$ parties proportionnelles a des séginents donnes. Bulletin de la Societs Mathematique de France, t. IV, avril 1876 .

Intorno alla dimostrazione di un teorema fond mentule della teoria de' poli e polari nella a Geometria Projettiva w del Prof. L. Cremona. Giornale di Ballaglini, vol. XIV, 1876.

Jung-Bertini-Saviotti. "Lezioni di Statica Grafica per Antonio Favaro ». Cenno crituco. Politecnico, vol. XXVI.

G. Jung (Mildno). Note relatuve $d$ deux theorèmes de Lagrange sur le centre de gravité. Bulletin de la Sociète Math. de France, t. VII, 1878 .

Elementi di Geometria Descrittıva del Dr. R. Sturm, traduzione dal tedesco. Hoepli, Milano, 1878 .

Sul problema inverso del momenti d' inerzia di una sezione piana. Soluzione grafica generale - Due note. Politecrico, anno XXIV, Milano.

Soluzione geomeccanica di alcuni problemi d'interpolazione. Rendiconti del. l' Istut. Lomburdo, is aprile i880.

Compensazione deglı errori proporzionali per un dato sistema di osservazioni dirette, ibid. 29 aprile 1880 .

Intorao al principio della medis arimetica. 'Politecnico, vol. XXIX, $s$ dicembre 1880 .

Sui momenti obliqui di un sistema di punti e sull' " imaginäres bild * di Hesse. Collectanea Mathem. in mem. Chelini, aprile $188 \mathrm{r}$.

Alcuni teoremi sulle forme degeneri dell'ellissoide del Culmann. Rendiconti dell' Ltit. Lombardo, 23 febbrajo $\mathbf{1 8 8 2}$.

Sul Pseudofoco del paraboloide e sul centro magnetico, ibid. is giugno 1882.

Alcuni teoremi baricentrici, tbud. 6 luglio 1882.

Osservazioni ed aggiunte alls nota precedente. sbid. 14 dicembre 1882.

Sui sistemi privi di baricentro, abid. 31 maggio 1883.

Nuovi teoremi a complemento della regola di Guldin e proprietd della spirale $r=a \frac{\operatorname{sen} \Theta}{\theta} . R$. Ace. dei Lincei, Transunti, vol. VII, 1883.

Sull'equilibrio dei poligoni articolati in connessione col problema delle con. figurazioni. Annali di Matcmatica, serie 2" t. XII, Milano 1884.

Sopra una classe di configurazioni d'indice 3. Rondiconti dell' Istituto Lombardo, 12 febbrajo 1885 .

Di alcune proprietd geometriche, statiche e cinematiche dei poligoni artico. lati, ibid. I2 marzo 1885.

F. Wloin (Leipzig). Zur Theorie der elliptischen Functionen ntez Stufe. Konigl. Suchs. Gesellschajt der Wissenscisaften, 14 november 1884.

G. Malsano (Palermo). Sistemi completi dei primi cinque gradi della forma ternaria biquadratica e degl'invarianti, covarianti e controvarianti di sesto grado; Giorn. di Butt, vol. XIX. 
G. Maisano (Palermo). Sulld forma binaria di quinto ordine. $R$. Ace. dei Lincei, Memorie, vol. XIV, 7 gennajo 1883.

Sopra due classi di forme binarie, ibill. XV, 18 marzo 1883.

La sestica binuria, ihil. XIX, 3 febbrajo 1884 .

A. Mannhoim (Paris). Quelques rísult uts obtenus p ir $/$ considération du díplacement infiniment petit d'une surface algébrique. Comptes Rendus, mai 1870 .

Détermination du plan osculateur et du rayon de courbure de la trajectoire d'un point quelconque d'une droite que l'on déplace en l'.tssujetissant $\downarrow$ certaines conditions, ibid., juin 1870 .

Construction de l'axe de courbure de la surface díveloppable enveloppe d'un plın dont le díplacement est assujetti 1 certaines eonditions, ibill. juin 1870 .

Générulisation du, théoreme de Meusnier, ibid. février 1872 .

Détermination de la liaison géométrique qui existe entre les éléments de la courbure des deux nappes de la surface des centres de courbure principaux d'une surface donné, ibrd. fivrier 1872 .

Recherches géométriques sur le contact du $3^{e}$ ordre de deux surfaces, ibid. murs 1872.

Construction directe du r.ayon de courbure de la courbe de contour apparent d'une surface qu'on projette orthogonalement sur un plan, ibid. avril 1874 .

Détermination des relutions an.llytiques qui existent entre les śléments de courbure des deur nappes de la développée d'une surface, ibid. décembre 1874 .

Sur les surfaces tr.jectoires des points d'une figure de forme invariable dont le déplacement est assujetti $d$ quatre conditions. Journ. de SCath. 3* série, t. I, 1875 .

Solutions géométriques de quelques problèmes, relatifs $\mathfrak{a}$ la thíorie des surfaces, et qui dipendent des infiniment petits du $3^{\text {tme }}$ ordre, Comples Rendus, mars 1875 .

Solutions géometriques de nouvedux problemes relatifs $d$ la theorie des surfaces et qui dependent des infiniment petuts du $3^{\text {twe }}$ ordre, ibid., mars', 875 .

Note d l'occusion de la Communication fatte par M. Ribaucour dans la séance du is mars 1875 , ibid., 22 nars 1875.

Nouvelles propriétés géométriques de la surface de l'onde, qui s'interpretent en Optique, ibıd., 7 février 1876 .

Démonstration géométrique d'une relation due a M. L aguerre, ibid., 6 mars 1876 .

Construction pour un point de $1 d$ courbe d'intersection de deux surfaces du centre de la sphère osculatrice de l$\perp$ courbe, 2 bid., 27 novembre 1876 .

Sur le paraboloide des huit droites, ibid.. 2 avril 1877 .

Sur les surfaces dont les rayons de courbure principsux sont fonctions l'un de l'autre, ibid., 30 avril 1877 . 
4. Mnenhoim (Paris). Sur le deplacement infiniment petit d'un diedre de grindeur invariable; deux notes, Comptes Rendus, II juin, 23 juillet 1877 .

Sur les plans tangents singuliers de la surface de l'onde et sur les sections faites dans cette surface par des plans piralleles 2 ces plans tangents. Assoc. franc. pour l'avanc. des Sciences, Congres du Havre, 24 aod 1877.

Sur la surface de l'onde, ibid., 25 asat 1877.

Sur les normales de la surface de l'onde, ibid., 27 aoat 1877.

Nouveau mode de représentation plane de classes de surfaces réglées; trois notes. Comptes Rendus, 29 octobre, S, 19 novembre 1877 .

Sur les surfaces réglées. Journ. de Math. 3 " série, t. IV, février 1878 .

De l'emploi de la courbe reprèsentative de la surface des normales principales d'une courbe gauche pour la démonstration de propriétés relatives à cette courbe. Comptes 'Rendus, 20 mai 1878 .

Sur la surface de l'onde. Ass. frans. pour l'av. des Sciences, Congris de Paris, 24 aout $r 878$.

Transformation par polaires réciproques d'un pinceau de normales et extensions, ihid., 27 aout 1878 .

Construction de la normale a la surface trajectoire d'un point d'une figure de forme invariable dont le déplucement est assujetti à quatre conditions, ibid., 28 aodt 1878 .

Construction des centres de courbure principaux de la surface de vis a filet triangulaire, ibid., 29 aoút $1 \$ 78$.

Construire les axes d'une ellipse, étant donnis deux di.umètres conjugués. Nouvelles Annales de Math., 2e série, t. XVII, 1878.

Détermination géométriçue des ombilics de la surface de l'onde. C. $R$., ; $m i s$ r 879 .

Constructions planes des éléments de courbure de la surface de l'onde. Extratt du volume in memorian $D$. Chelsni, juillet 1879 .

La surface de l'onde considérée comme surface limite. $C$. $R$., 26 avril 1880. Nouvelle générution de la surface de l'onde et constructions diverses, ibid., 7 juin 1880 .

Notice sur les travaux geométriques de A. Mannheim. Paris, 1881 .

Sur la determination, en un point d'une suface du second ordre, des dxes de l' indicatrice et des rayons de courbure principaux. Journ. de Math., $3^{\text {e }}$ série, t. VIII, mai I 882 .

0. Masoni (Napoli). Sopra alcune curve del quarto ordine dotate di punti d'ondulazione. R. Acc. di Napoli, 4 febbrajo i 882.

Sui connessi conici ed in' particolare sui sistemı di rette del $2^{\circ}$ ordine , sbid., aprile 1883 .

Sull'urto dei corpi e sal movimento d'un corpo pesante fra due mezzi resistenti, shid., marzo 1884 .

Sulle derivate d'ordine qualunque della funzione potenziale quando l'dttrazione é proporzionale all' inverso della $n^{\text {ma }}$ potenza della distanza, ibid., 14 guugno 1884 . 
0. Masoni (Napoli). Sulle forze impulsive che hanno la medesima axione sopra uno stesso punto di un sistema rigido, $R$. Acc. di Napoli, luglio 1884.

G. Hittag-Lefler (Stockholm). Sur la representation analytique des fonctions monogenes uniformes d'une variable indépendante. Acta Mathematica, IV.

M. Noether (Erlangen). Zur Theorie der algebraischen Functionen mehrerer complexer Variabeln. Götting. Nacisricbten, 1869.

Zur Theorie des eindeutugen Entsprecluens algebraischer Gebilde von beliebig vielen Dimensionen, Math. Annalen, Band II, 1870.

Ueber Flachen, welthe Scharen rationaler Curven besitzen, ibid., III, 1871.

Sulle curve multiple di superficie algebriche. Annall di Matematica, serie $2^{\circ}$, t. $V, 3871$.

Rudolf Friedrich Alfred Clebsih. Versuch einer Darlegung und Würdigung seiner wissenschaftlichen Leistungen von einigen seiner Freunde. Math. Annalen, Band VII, 1873.

Ueber die algebraischen Functionen und ihre Anwendung in der Geometrie. Zus. mit Brill, ibid., VII, 1874 .

Zur Theorie des endeutigen Entsprechens algebraischer Gebilde. Zweiter Aufsatz, ihad., VIII, 1875 .

Otto Hesse. Zettschrift fur Math. und Physik von Schlömilch, 20 Jahrgang 1875 .

Ueber die algebraschen Formen mit identisch verschwindender Hesse 'scher Determinante. Sitzungsb. physik. medic. Societal zu Erlangen, 1876.

Zur Elimınationstheorse. Math. Ann., XI, 1877.

Zur Theorie der Thetafunctionen von vier Argumenten, wbid., XIV, 1879. Notiz über eine Classe symmetrischer Deteminanten, sbıd., XV1, 1880.

Ueber die invariante Darstellung algebrasscher Functionen, zbid., XVII. 1880.

Ueber einen Satz aus der Theorie der algebraischen Functionen. Journal für : Kathematık, Bd. XCII, $188 \mathrm{I}$.

Note über dıe algebraischen Curven, welche eine Schasr eindeutiger Transformationen in sich zuldssen. OCuth. Ann, XX, 1882 .

Nachtrag zur " Note uber die algeb. Curven mit einer Schadr eindeutiger Transformationen in sich. $"$, ibrd., XXI, 1883.

A. Pepoll (Palermo). Sopra un problema delle trasformazioni Cremoniane. Atti del Collegio degli Ingegmeri ed Arch. di Palermo, 1884.

G. Pittaluga (Palermo). Degli assi elastici. Atti della $R$. Acc. delle Scienze di Torino, XIV, 6 aprile 1879 .

P. H. Schoute (Groningen). Homographie et son application $\mathrm{d}$ la theorie des surfaces du seennd ordre. Arebives Nierlandasses, t. VI, 1871 .

Over eene bijzondere ruimtekromme van den zevenden gradd.

De la projection sur une surface. Assoc. franc pour l' avanc. des Sciences, Congres de Montpellier, 1879.

Sur les courbes tracees sur une surface du second ordre, ibid.

Sur ld transformation conjuguée, tbid. 
Bohouto-Dowrulf. Déterminer une courbe unicursale de $4^{\text {tme }}$ ordre ayant des points doubles en $A_{1}$ et $A_{2}$, et passant par les sept points $I^{\prime}, 2^{\prime}, 3,4,5,6$ et 7 , tbid.

P. B. Sohoute (Groningen). Sur une transformation géométrique et sur la généralisation d'un problème de la théorie des enveloppes dites «Courbes de sureté v. Congrits de Reims, 1880.

De la transformation conjuguée dans l'espace, ibid.

De Kegelsneden in de projectivische meetkunde. Groningen, $188 \mathrm{I}$.

Deux cas particuliers de la transformation birationnelle. Bulletin de Darboux, 2e série, t. VI, 1882 .

Die Steinerschen Polygone. Journal de Crelle, 95, 1883.

Nachtrag zur Abhandlung "Die Steinerschen Polygone ", ibid.

Application de la transformation par droites symetriques $d$ un probleme de Steiner. Bulletin de Darboux, $2^{e}$ serie, t. VII, 1883.

Sur deux transformations géonétriques uniformes. Congrès de Rouen, 1883 .

Nachtrıg zur Abhandlung " Die Steinerschen Polygone, Crelle, 95.

Notiz über die Lemniscate, Sizzb. der Wiener Akud. der Wissensch., 1883.

Finige Bemerkungen über dis Problem der Glanapunkte, ibid.

Over een bijzondere kromme van den vierden graad met drie dubbelpunten. K. Akad. van Wetensch., Amsterdam, 1884 .

Quelques théorèmes géomitriques. Congris de Blots, 1884 .

Sur $l_{1}$ construction de courbes unicursales par points et tungentes. Archives Nderlandaises, t. XX.

Scuola di Applicazione per gl'INGegneri di Roma. Catalogo dell i Biblioteca. Supplementi $1^{\circ}, 2^{\circ}, 3^{\circ}$.

G. Torelli (Napoli). Sulle funzioni simmetriche complete e semplici. Grorn. di Batt. vol. $\mathrm{V}, 1867$.

Di alcuni integrali formati dagl' integrali ellittici e di qualche loro applicacazione, ibid. $\mathrm{XI}, 1873$.

Moltiplicazione grafica delle rette e trasformazone grafica delle figure piane, etc. Napoli, 1875 .

Sei lezioni di geometri، descrittiva. Napoli 1877 .

Sopra alcune proprietม́ numeriche. Giorn. di Batt., XVI, 1878.

Versione dal tedesco di una nota di G. Fiedler: Sulla riforma dell' inseguamento geometrico, seguita da tre lettere inedite dell' autore, ibid. $\mathrm{XVI}, 1878$.

Commemorazione dr G. Bellavitis. Atti dell'Acc. Pontaniana.

Sui determinantı circolanti. R. Acc. di Napoli, aprile 1882.

Commemorazione di N. Trudi. Atte dell' Acc. 'Pontamana, vol. XVI.

Tre lezioni di geometria elementare. Napoli 1884.

Un problemd sulle espression differenziali, Annal de Matem., serie 2*, t. XIII, I884.

Collezione di sedıci memorie e note di diversi Auton. 


\section{4}

a. Btopharos (Athénes). Mémoire sur la thérie des formes binaires of cur f'ello mination. These presentse d la Faculte des Scionces de Paris, Juillet 1884 .

V. Volterra (Pisa). Sopra alcuni problemi della teoria del potenziale. Pisa 1883. Sulle figure elettrochimiche di $\mathrm{A}$. Guébh ard. Atti della $R$. Ace. \&l Tor rino, vol. XXIU, is febbraro 1883 .

Sopra un problema di elettrostatica. $R$. Acc. doi Lincei, Transunti, is gilugno 1884 . 


\title{
SEDUTA DEL S APRILE I885
}

\author{
PRESIDENZA G. ALBEGGIANI
}

\section{Comunicazioni :}

A. Capelli osserva che il teorema enunciato nella nota del sig. Hermite: Sur les fonctions bolomorphes (") non deve considerarsi come nuovo, ma che solo si da di esso una nuova dimostrazione. Esso puó ricavarsi, con dimostrazione semplice, dal teorema, richiamato dallo stesso Hermite, che una funzione olomorfa il cui modulo resta finito per ogni valore della variabile è una costante. Un teorema analogo, più generale, di cui questo è conseguenza, fu dimostrato da Schwarz nella Memoria : Ueber die Integration der Differentialgleichung $\Delta^{2} u=0$ (Borchardt's Journal, Bd. 74), cioè, che una funzione $u$ la quale nel$l$ ' interno di un cerchio di raggio $R$, grande quanto si vuole, soddisfa all'equazione $\Delta^{2} u=0$ ed è monodroma finita e continua insieme alle sue prime derivate, ed ha le secunde derivate finite, e la quale inoltre sin tale che il suo valore per quanto cresca $R$ resta inferiore in valore assoluto ad una grandezza finita $g$ (indipendente da $R$ ), è una costante. Lo stesso Autore conclude che analogamente si puó dimostrare che, cssendo $u(r, \varphi)$ l'espressione di $u$ in coordinate polari, se $\frac{u(r, \varphi)}{r^{n}}$ resta finita in tutto il piano, oltre a soddisfare alle solite condizioni, allora $u(r, \varphi)$, considerata come funzione di $x$ e di $y$, è un polinomio intero di grado $n$ in $x$ ed in $y$.

Questi teoremi si estendono immediatamente .d un numero qualunque di variabili.

(*) Journal de Jordan, $I_{4}, 1885$. Vedi « Rivista bibliografics " seduta 22 mar20188 s (p. 28). 


\section{SEDUTA DEL I 9 APRILE $188 \mathrm{~s}$}

PRESIDENZA G. ALBEGGUNI

\section{Comunicazioni :}

P. Gambera ritorna sulla coerenza dei corpi e sulle formole

$$
\frac{k}{k^{\prime}}=\frac{c d \delta^{\prime}}{c^{\prime} d^{\prime} \delta}, \frac{P c t}{p^{\prime} d^{\prime} t^{\prime}}=\frac{P k d^{\prime} \delta t}{p^{\prime} k^{\prime} d \delta^{\prime} t^{\prime}}=\frac{k \frac{P}{d} \delta t}{k^{\prime} \frac{P^{\prime}}{d^{\prime}} \delta^{\prime} t^{\prime}}-\frac{k v \delta t}{k^{\prime} v^{\prime} \delta^{\prime} t^{\prime}}
$$

mostrando la relazione fra la coerenza e la dilatabilith termica dei corpi e le calorie di riscaldamento necessarie per produrre un certo aumento di temperatura.

\section{SEDUTA DEL Io MAGGIO I 885}

PRESIDENZA G. ALBEGGIAN1

Comunicazioni :

G. Maisano comunica alcune sue ricerche sul gruppo di punti situati sull Hessiana di una curva di ordine $n$, le cui tangenti toccano la Cayleyana; trova che siffatto gruppo costituisce la completa intersezione dell'Hessiana colla curva dell'ordine $8 n-18$ :

$$
V \equiv(a b H)\left(a b H^{n}\right) a_{x}^{a n-2} b_{x}^{n-2} H_{x}^{p m-1} H_{x}^{3 n-7}=0
$$

ove $a_{x}^{*}-b_{x}^{n}=c_{x}^{*}=\ldots=0$ rappresenta simbolicamente la curva fondamentale e $H_{x}^{3 n-6}=H_{x}^{3 n-6}=\ldots=0$ la sua Hessiana.

Pel caso particolare che la curva fondamentale sia del $t^{\circ}$ ordine, perviene alla curva del $14^{\circ}$ ordine :

$$
2 H_{9} H_{\theta}^{\prime} H_{x}^{S} H_{x}^{S} \Theta^{4}-\varsigma \theta_{x}^{4} H_{9}^{2} H_{x}^{4} H_{x}^{16}=0
$$


ove è postu

$$
\Theta_{x}^{4} u_{\theta}^{2}-(a b u)^{2} a_{x}^{2} b_{x}^{2}
$$

La curva (2) che taglia sull' Hessiana i 6.14 punti, le cui tangenti toccano la $\mathrm{C}_{a}$ yleyana, è la stessa curva che sega la curva fondamentale $f=0$ nei punti di contatto delle sue tangenti doppie, come risulta dal calcolo delle stesso Autore, fondato sopra i risultati ottenuti nella di lui Memoria Sui sistemi compleli dei primi cinque gradi, etc. pubblicata nel Giornale di Ballaglini, vol. XIX.

Proposta di quesiti:

G. B. Guccia legge il seguente problema comunicatogli, con lettera privata, dal Prof. P. H. Schoute, dell'Università di Groninga :

On donne une surface quadrique $\mathrm{S}$ à centre $\mathrm{C}$ et un plan $\mathrm{P}$ perpendiculaire à un des axes $\mathrm{AA}^{\prime}$ de la surface au point milieu $\mathrm{B}$ de $\mathrm{CA}^{\prime}$. Si d'un point quelconque de l'espace represente par $\mathrm{M}_{2 n}$ on parvient au point $\mathrm{M}_{2 n+1}$ correspondant, en cherchant sur le droite $\mathrm{CM}_{2 n}$ le point conjingui it $\mathrm{M}_{2 n}$ par rapport à $\mathrm{S}$, el d'un point quelconque $\mathrm{M}_{2 n+1}$ au point $\mathrm{M}_{2 n+2}$ correspondant, en prenant le point symlirique de $\mathrm{M}_{2 n+1}$ par rapport aे $\mathrm{P}$, on dernande il demontrer que $\mathrm{M}_{m+6} \equiv \mathrm{M}_{m}$ pour chaque point $\mathrm{M}_{m}$ de lespidee et de chercher le lien des points pour lesquels $\mathrm{M}_{m+2} \equiv \mathrm{M}_{m}$, pour lesqutels $\mathrm{M}_{2 n+3} \equiv \mathrm{M}_{2 n}$ ou pour lesquels $\mathrm{M}_{2 m+4} \equiv \mathrm{M}_{2 n+1}$.

SEDUTA DEL 17 MAGGIO 188 ;

PRESIDENZA P. GAMBERA

Comunicazioni :

F. Cavallaro espone alcuni suoi studi sopra una corrispondenza birazionale d'ordine $n$ fra due spazi a tre dimensioni, da lui ottentata con particolare procedimento di ripetizione di una stessa trasformatione quadratica. 


\title{
$4^{8}$
}

G. B. Guccia aggiunge brevi osservazioni.

A. Capelli si occupa della soluzione del problemal del Professor Schoute, letto nella seduta precedente dal Dr. Guccia.

$\mathrm{Ne}$ segue discussione a cui prendono parte i soci Pepoli e Guccia; in scguito alla quale il socio Capelli si riserba di ritornare sull'argomento.

\section{SEDUTA DEL 3 I MAGGIO I885}

\author{
PRESIDENZA G. MAISANO
}

Comunicazioni :

A. Capelli ritoma sulla soluzione del problema del Professore Schoute (vedi seduta precedente) e lo formula nei termini seguenti :

Se si indica con $\Omega$ la trasformazione piana per cui da un punto $P$ si passa al punto $\mathrm{P}^{\prime}$, coniugato di $\mathrm{P}$ rispetto ad una conica fissa $\mathrm{C}$, sopra la retta che congiunge $\mathrm{P}$ col centro della conica, e con $\Omega^{\prime}$ la trasformazione dello stesso piano per cui da un punto $\mathrm{P}$ si passa ad un punto $\mathrm{P}^{\prime}$, simmetrico di $\mathrm{P}$ rispetio ad una retia parallela ad uno dei due assi della conica e passante pel punto di mezzo dell'altro semi-asse; se ad un punto $\mathrm{M}_{1}$ qualsivoglia si applicano successivamente le 6 trasformazioni $\Omega, \Omega^{\prime}, \Omega, \mathbf{2}^{\prime}, \Omega, \Omega^{\prime}$, si dedurranno da $\mathrm{M}_{1}$ altri 6 punti $\mathrm{M}_{2}, \mathrm{M}_{3}, \mathrm{M}_{4}$, $\mathrm{M}_{5}, \mathrm{M}_{6}, \mathrm{M}_{7}$, successivamente, l'ultimo dei quali $\mathrm{M}_{7}$ coincide semtre col punto di partenza $\mathrm{M}_{1}$.

Per venire alla soluzione del detto problema comincia dallo esporre alcune considerazioni generali sopra i problemi geometrici che ammettono un numero infinito di soluzioni.

Osserva in particolare che in Geometria si possono enunciure alcuni problemi per modo che essi non ammettono alcuna soluzione, ovvero ne ammettono un numero infinito: nel mentre che questi stessi problemi espressi algebricamente darebbero luogo in generale ad un numero tinito $k$ di soluzioni. Ciò invero decade perché il problema geo- 
metrico è di natura tale da ammettere sempre $k$ soluzioni intproprie, le quali esauriscono le $k$ soluzioni date dall'Algebra, quindi se esse sono tali da ammettere almeno ancora una soluzione propria, il corrispondente problema algebrico ammetterebbe cosi $k+$ I soluzioni, cioè almeno una più del grado dell' equazione risultante a cui esso conduce. Tale equazione sar.l dunque soddisfatta identicamente ed il problema algebrico avrà quindi infinite soluzioni. Cosi p. e. il teorema che « se esiste un poligono di $n$ lati inscritto in una conica e circoscritto ad una altra data, ne esistono infiniti " è una conseguenza immediata di questo principio.

Consider.l separatamente $\mathrm{i}$ casi di problemi posti in un campo di numeri o di punti semplicemente infinito, ovvero in un campo di numeri o di punti doppiamente infinito, quale p. c. il piano. In quest'ultimo caso mentre si puó asserire, che, ammettendo il problenra sempre $k$ soluzioni improprie ed almeno una soluzione propria, ammettera infinite soluzioni, non si potril d'altra parte dire, senz'altro, che esso ammette un numero doppiamente infinito di soluzioni; cioè che ogni pinto del piano soddisferd alle condizioni imposte dal problema. A tale oggetto occorrono altri criterì, uno dei quali dimostr.l potere essere il seguente :

Siano

$$
f_{m}(x, y)=0, \quad \varphi_{n}(x, y)=0
$$

le dice equazioni in $x, y$ risp. di grado $m$ ed $n(m>n)$ che servono a determinare le coordinate $x, y$ dei $k$ punti del piano soddisfacenti alle condizioni del problema. Allora se le condizion del problema sono verificate $d a(m+1)^{2}$ punti del piano che siano l'ntersezione completa di un fascio di $m+\mathrm{I}$ rette distinte con un fascio di altre $m+\mathrm{I}$ rette distinte, esse saranno verificate certamente da tutti i punti del piano.

Ove poi il problema conduca ad un. corrispondenza o trasformazione piana $\left(P_{m}, P_{n}^{\prime}\right)$ che ad ogni punto $P(x, y)$ fa corrispondere uno o più punti $P^{\prime}\left(x^{\prime}, y^{\prime}\right)$ e reciprocamente, e possa condursi alla determinazione dei punti-uniti, allora, se la trasform.zzione è tale da permettere di accertare facilmente l' esistenzal di curve 1 cui punti sono tutti uniti, basterh dimostrare l'esistenzal di una o piu curve siffatte il cui ordine complessivo superi $n^{\prime}$, essendo $n^{\prime}$ l'ordine della curva che cor- 
50

risponde ad una retta della $I^{2}$ figura, pex potere asserive che tutti i pund del pimo sono uniti. (Continua, vedi seduta seguente).

C. B. Guccia. Formole analitiche per la trasformazione Cremomiana:

$$
\text { (n dispari) } \quad x_{1}=3, \quad \alpha_{2}=n-2, \quad a_{n-3}=1 .\left(^{*}\right) \text {. }
$$

Sia $n=2 \mu+1$. La rete omaloidica é formata da curve d'ordine $2 u+1$, le quali hanno un punto $(2 \mu-1)$-plo, $2 \mu-1$ punti doppi e tre punti semplici fondamentali. Posto per brevitd :

$$
U=x_{3} u_{\mu-1}+u_{\mu}, \quad V=x_{3} v_{\mu-2}+v_{\mu-1},
$$

dove $u_{\mu_{1}}, u_{\mu_{1}}, v_{k_{1}}, v_{\mu_{1}}$ indicano polinomi omogenei, rispettivamente dei gradi $\mu, \mu-1, \mu-I, \mu-2$, nelle variabili $x_{1}, x_{2}$; si hanno per l'attuale trasformazione le fornole seguenti :

$$
\begin{aligned}
& \rho y_{1}=\left(U+\alpha x_{1} V\right)\left[U\left(x_{1}+x_{2}\right)+x_{1} x_{2} V\right] \\
& \rho y_{2}=\left(U+\beta x_{1} V\right)\left[U\left(x_{1}+x_{2}\right)+x_{1} x_{2} V\right] \\
& \rho y_{3}=x_{2}\left(U+\alpha x_{1} V\right)\left(U+\beta x_{1} V\right) .
\end{aligned}
$$

Infatti le curve del piano $(x)$ corrispondenti alle rette

$$
\lambda_{1} y_{1}+\lambda_{2} y_{2}+\lambda_{3} y_{3}=0
$$

del piano $(y)$ sono dell'ordine $2 \mu+\mathrm{I}$, e passano:

$I^{\circ}$ con $2 \mu-I$ rami pel punto

$$
x_{1}=x_{2}=0
$$

$2^{\circ}$ con due rami pel punto di ulteriore intersezione delle linee

$$
x_{1}=0, \quad U=0
$$

() Cremon a, Bulletin de Darboux, $V_{1}$, p. 224 . 
e parimenti con due rami per ognuno dei

$$
\mu(\mu-1)-(\mu-1)(\mu-2)=2(\mu-1)
$$

punti di ulteriore intersezione delle curve

$$
U=0, \quad V=0 ;
$$

$3^{\circ}$ con un ramo per ognuno dei punti di ulteriore intersezione delle linee:

$$
\begin{aligned}
x_{2} & =0, & U-0 ; \\
U+\alpha x_{1} V & =0, & U\left(x_{1}+x_{2}\right)+x_{1} x_{2} V=0 ; \\
U+\beta x_{1} V & =0, \quad & U\left(x_{1}+x_{2}\right)+x_{1} x_{2} V=0 .
\end{aligned}
$$

Formole analitiche per la trasformazione Cremoniana:

$$
a_{1}=\frac{2 n-8}{3}, \quad \frac{x_{n-1}}{3}=3, \quad \frac{a_{n+2}}{3}=2, \quad \frac{a_{2(n-1)}}{3}=1 .\left(\left(^{*}\right)\right.
$$

Sia $n=3 \mu+1$. La rete omaloidica e formata da curve d'ordine $3 \mu+1$, le quali hanno : un punto $2 \mu$-plo, due punti $(\mu+1)$-pli, tre punti $\mu$-pli $\mathrm{c}_{2}(\mu-1)$ punti semplici fondamentali. Poniamo :

$$
U=s_{3} u_{\mu_{1}}+u_{\mu}, \quad V=s_{1} v_{\mu_{-1}}+v_{\mu_{1}},
$$

dove $u_{\mu}, u_{\mu_{1}}, v_{\mu_{1}}, v_{\mu_{2}}$ sono polinomi omogenei, rispettivamente dei gradi $\mu, \mu-1, \mu-1, \mu-2$, nelle $s_{1}, s_{2}$, mentre

$$
\begin{aligned}
& s_{1} \equiv t_{1}\left(t_{2}+t_{1}\right) \\
& s_{3} \equiv t_{3}\left(t_{3}+t_{1}\right) \\
& s_{3} \equiv t_{3}\left(t_{1}+t_{2}\right)
\end{aligned}
$$

(") Cremona, Bullatin de Darboux, V1, p. 227. 
dove $^{52}$

$$
\begin{gathered}
t_{2}+t_{3} \equiv x_{1} x_{3} \\
t_{3}+t_{1} \equiv x_{2} x_{1} \\
t_{3} \equiv a x_{2} x_{3}+b x_{3} x_{1}+c x_{1} x_{2} .
\end{gathered}
$$

Se ora scriviamo l'equazione

$$
\lambda_{1} U+\left(\lambda_{2} s_{3}+\lambda_{3} s_{2}\right) V=0
$$

ed eseguiamo in essa le precedenti sostituzioni, \& facile riconoscere che dal primo membro si staccherd il fattore $x_{3}^{\mu-1}$. Dico che il fattore residuale posto uguale a zero, della form.l

$$
\lambda_{1} K+\lambda_{2} L+\lambda_{3} M=0,
$$

rappresentert l'equazione della rete omaloidica data.

Infatti ognuna di queste curve è dell'ordine $3^{\mu}+\mathrm{r}$, e passa :

$I^{\circ}$ con $2 \mu$ rami pel punto

$$
x_{1}=x_{2}=0 ;
$$

$2^{\omega}$ con $\mu+$ I rami pei due punti

$$
x_{2}=x_{3}=0, \quad x_{3}=x_{1}=0 ;
$$

$3^{\circ}$ con $\mu$ rami per ognuno dei tre punti in cui le coniche

$$
t_{1}=0, \quad t_{2}=0, \quad t_{3}=0
$$

$s$ 'incontrano ulteriormente due a due;

$4^{\circ}$ con un ramo per ciascuno dei

$$
\begin{array}{r}
(3 \mu+1)(3 \mu-2)-2 \mu(2 \mu-2)-2 \mu(\mu+1)-3 \mu(\mu-1) \\
-2(\mu-1)
\end{array}
$$


punti di ulteriore intersezione delle curve residuali

$$
U^{\prime}=0, \quad V^{\prime}=0
$$

Si hanno cosi le formole di trasformazione :

$$
y_{1}: y_{2}: y_{3}=K: L: M
$$

\section{SEDUTA DEL I 4 GIUGNO I 885}

PRESIDENZA G. ALBEGGIANI

Ammissione di nuovi soci: Dr. Andrea Cantone.

Comunicazioni :

A. Capelli applica l'ultimo dei metodi generali da lui esposti nella seduta precedente, alla dimostrazione del teorema del sig. Schoute (p. 48). Mantenendo le stesse notazioni, dimostra che la trasformazione univoca $\Theta$, risultante dalle sei trasformazioni

$$
\Omega, \Omega^{\prime}, \Omega, \Omega^{\prime}, \Omega, \Omega^{\prime},
$$

applicate successivamente, ¿̀ una trasformazione identica.

Evidentemente la trasformazione $\Theta$ dovrh essere una trasformazione univoca (Cremoniana) al massimo dell'ottavo ordine, poichè essa consta di tre trasformazioni di $2^{\circ}$ ordine e di tre di $I^{\prime \prime}$ ordine.

Pertanto, per dimostrare che la trasformazione $\theta$ e identica, cios che tutti i punti del piano sono punti uniti, basterd dinostrare l' esistenza di un complesso di curve i cui punti siano tutti uniti, ed il cui ordine complessivo sia superiore ad 8 , cioè almeno eguale a 9 .

Il prof. Capelli raggiunge tale intento dimostrando con consi- 


\section{4}

derazioni geometriche semplicissime l'esistenza di quattro rette speciali e di tre curve speciali del second' ordine (una delle quali è la conica stessa del teorema) i cui punti sono tutti uniti.

G. Maisano. Sui covarianti indipendenti di $6^{\circ}$ grado nei coefficienti della forma biquadratica ternaria. (*).

Premette i seguenti teoremi :

I. Dato un gruppo equianarmonico di 4 elementi rappresentanti una forma binaria biquadratica, il gruppo Steineriano coincide col gruppo dato.

II. Le quattro tangenti che da un punto $P$ della curva di $4^{\circ}$ ordine $f=0$ si possono condurre allo inviluppo

$$
(a b u)^{4}=u_{\alpha}^{4}=u_{\beta}^{4}=u_{\gamma}^{4}=\ldots=0,
$$

sono le quattro tangenti che dal punto $P$ si possono condurre alla cubica polare di $P$ rispetto ad $f=0$.

III. Si hanno sulla curva $f=0$ I 6 punti, intersezioni della curva $f=0$ colla curva

$$
S=(a b c)(a b d)(a c d)(b c d) a_{x} b_{x} c_{x} d_{x}=0,
$$

le cui cubiche polari rispetto alla stessa curva $f=0$ sono equianarmoniche; e 24 punti, intersezioni della curva $f=0$ colla curva

$$
T=(a b c)(d e f)^{2}(a b d)(a c e)(b c f) a_{x} b_{x} c_{x} d_{x} e_{x} f_{x}=0,
$$

le cui cubiche polari rispetto ad $f=0$ sono armoniche.

IV. Le quattro tangenti che da un punto del piano si possono condurre alla curva

$$
(a b u)^{4}=u_{x}^{4}-u_{\beta}^{4}=u_{r}^{4}=\ldots=0
$$

(") Cfr. G. Maisano: Sistemi completi dei primi cinque gradi, etc. (Giomale di Battaglini, vol. XIX). 
formano un gruppo equianarmonico od armonico se il punto giace, rispettivamente, sulla curva

$$
(\alpha \beta x)^{4}=0,
$$

ovvero sulla curva

$$
(\alpha \beta x)^{2}(\alpha \gamma x)^{2}(\beta \gamma x)^{2}=0
$$

Da questi teoremi segue che la curva $S$ deve appartenere al fascio

$$
x A f+\lambda(\alpha \beta x)^{4}=0
$$

ove $A-(a b c)^{4}$ è l'invariante di $3^{\circ}$ grado della forma fondamentale; ed infatti nella citata Memoria trovasi :

$$
S=\frac{1}{4}(\alpha \beta x)^{4}-\frac{1}{3} A f
$$

e che la curva $T$ deve appartenere al fascio

$$
x \cdot(\alpha \beta x)^{2}(\alpha \gamma x)^{2}(\beta \gamma x)^{2}+\lambda \cdot C f=0,
$$

in cui $\mathrm{C}$ è un covariante di $2^{\circ}$ ordine e di $5^{\circ}$ grado e quindi della forma

$$
\mu C_{1}+v C_{2}
$$

indicando con $C_{1}$ e $C_{2}$ i due covarianti di $2^{\circ}$ ordine e di $5^{\circ}$ grado della forma biquadratica. Si trova iafatti

$$
(\alpha \beta x)^{2}(\alpha \gamma x)^{2}(\beta \gamma x)^{2}+8 T=\frac{2}{25} f\left[63(\alpha \beta x)^{2} a_{\alpha}^{2} a_{\beta}^{2}-20 a_{\beta}^{2} a_{x}^{2}\right],
$$

servendosi della notazione : $(a b u)^{2} a_{\alpha}^{2} b_{\alpha}^{2}=u_{p}^{2}$. 
56

$\mathrm{Si}$ conchiude pertanto che $i$ covarianti indipendenti di $6^{\circ}$ grado sono solamente due e del 6 ordine.

G. B. Guccia espone alcune sue ricerche sulle Trasformazioni Cremoniane nel piano ("), dando implicitamente la soluzione del quesito da lui proposto nella seduta del 15 maggio 1884 (vedi pag. 5). Sia $n$ l'ordine della trasformazione e $\rho$ il numero dei punti fondamentali in ognuna delle figure. Sia inoltre, nel piano:

$R$ il punto le di cui isologiche sono dotate d'unil cuspide;

$D$ il punto le di cui isologiche sono dotate di due punti doppi;

I la retta luogo d'un punto le di cui isologiche toceano lit retta istessa secondo un contatto di second' ordine (rispettivamente in due punti fissi $i, i^{\prime}$ ), ossia : la relta che $\dot{i}$ langente d'inflessione (rispettivamente in due punti $\left.i, i^{\prime}\right)$ delle curve che ad essa corrispondono nella it a nella $2^{a}$ figura.

Tla retta luogo d'un punto le di cui isologiche toccano due volte la retta istessa (rispettivamente in due coppie di punti $t_{1}, t_{2} ; t_{1}, t_{2}$ ), ossia : la retta che é tangente doppia (rispettivamente in due coppie di punti $t_{1}, t_{2} ; t_{1}^{\prime}, t_{2}^{\prime}$ ) delle curve clse ad essa corrispondono nella $i^{\star} e$ nella $2^{*}$ figura.

Si hanno allora i teoremi seguenti :

I. La trasformazione Cremoniana ammeth, in generale:

$$
1^{\circ} \quad 24(n-1)
$$

punti $R$, cpperó altrettante coppie di isologiche dotate di cuspide;

$$
2^{0} n(17 n+6 p-63)+\frac{1}{2} p(p-7)+4^{6}
$$

puntl $D$, epperd altrettante coppie di isologiclse dotale di dise purti doppl;

$$
3^{\circ} \quad 18 n-3 p-27
$$

(") Cfr. Guecia: Sur les transformations Cremona dans to plan. (Comples Rendus, t. Cl, p. 866). 
rette $I$, epperò altrettante coppie di fasci (proiettivi) di isologiche, in 0 gnuna delle quali due curve corrispondentiqualunque banno, ciascuna, un contatto di second'ordine con una medesima relta, rispeltivamente in dice punti fissi ;

$$
4^{\circ} \quad 4[2 n(n-6)+p+13]
$$

rette $T$, epperd altrettante coppie di fasci (proiettivi) di isologiche, in ognuna delle quali due curve corrispondenti qualunque banno, ciascuna, un doppio contatto con una medesima retta, rispeltivamcnte in due coppie di punti fissi.

II. I punti $D, R$, le rette $T, I$ sono nel tempo istesso, rispeltivamente, punti doppi, cuspidi, tangenti doppie, tangenti di flesso, d'una curva, $\Theta$ dell'ordine $6 n+p-3$, della classe $4(n-1)$ e del genere $8 n-p-10$, cosi definita :

a) luogo del punto le di cui isologiche banno un punto doppio;

b) inviluppo della retta che tocca le curve che ad essa corrispondono nella $10^{*}$ e nella 2." figura. La curva $\Theta$ possiede inoltre un punto $(r+1)$-plo in ogni punto fondamentale $r$-plo di ciascuna delle figure, ed un punto doppio in ognuno degli $n+2$ punti uniti della trasformazione.

III. La curva $\Theta$ corrisponde punto a punto a ciascuna delle curve $J, I^{\prime}$ jacobiane delle reti (projettive) delle isologiche relative a tutti i punti del piano: Dato un punto $j$ (ovvero $j^{\prime}$ ) della curva $J$ (ovvero $f$ ), per esso passano infinite isologiche che ivi si toccano. La tangente comune è la retta $\overline{1 j^{T}}$, la quale e, nel tempo istesso, il liogo dei centri d'isologia delle coppie di isologiche che toccano, risp. in $j$, in $i^{\prime}$, la retta $\overline{j j^{\prime}}$. Fra i punti di questa retta ve ne ba uno, ed uno solo, $\theta$, appartenente alla curva $\Theta$, le di cui isologiche banno un punto doppio rispettivamente in $j$, in $j$. Nel punto $\Theta$ la retta $\bar{j} \bar{j}$ tocca la curva $\Theta$. Viceversa, dato un punto $\theta$ della curva $\Theta$ gli omologlti $j, j^{\prime}$, delle curve $J, J$, si oltengono quali punit doppl dalle isologiche di (a.

IV. Le tangenti alla curva $\Theta$ che possono condursi da un punto qualinque $p$ del piano, sono le retle che conginngono $p$ ai $4(n-1)$ punti di ulleriore intersezione della relativa isologica $P\left(P^{\prime}\right)$ colla curva $J\left(J^{\prime}\right)$, ovvero li rette che si possono condurre da $p$ a toccare altrove la relativa isologica $P\left(P^{\prime}\right)$. Etc. 


\title{
SEDUTA DEL 24 GENNAJO 1886 :
}

\author{
PRESIDENZA G. ALBEGGIANI
}

Elezione del Consiglio Direttivo per l'anno 1886 :

Dietro votazione a schede segrete vengono confermati in carica tutti i membri del Consiglio Diretivo. (Vedi sedute del 20 marzo 1884 ed I I gennaio I $885, p_{4}$ I e $I_{4}$ ).

A mmissione di nuovi socî: Dr. Vittorio Martinetti, Dr.|Francesco Giudice, Giovanni D'Arone.

Comunicazioni :

A. Capelli ranmentata l.a formola per lo sviluppo di una funzione di $n$ serie di variabili $n^{\text {are }}$ secondo le potenze del determinante delle variabili, moltiplicate per funzioni derivabili ds funzioni di un minor numero di serie di variabili (*), dimostra come una tunzione di piu di $n$ serie di variabili $n^{\text {are }}$ si possa derivare con operazioni invariantive, cioè con operazioni del tipo:

$$
D_{x y} \equiv y_{1} \frac{d}{d x_{1}}+y_{2} \frac{d}{d x_{2}}+\ldots+y_{n} \frac{d}{d x_{n}}
$$

da funzion di sole $n$ serie di variabili.

Detto poi numero delle funzioni di gradi dati nelle serie di variabili clse soddisfano a certe proprieta, il numero delle funzioni linearmente indipendenti, aventi tali gradi che possono derivarsi per mezzo di operazioni invari.untive dalla funzione piu generale avente tali gradi e soddi-

(7) Cír. Capelli : Fondaments di una beoru generale delle forme algebriche-(Memorie Acc. Lincei, XII, I882). 
sfacente a tali proprieta, dimostra che il numero delle funzioni di gradi dati di $n$ serie di variabili $n^{\text {arre }}$ derivabili con operazioni invariantive da funzioni di un minor numero di variabili, i uguale a quello di tutte le funzioni, degli stessi gradi, di $n$ serie di variabili $(n-1)$ arue.

IM. L. Albeggiani stabilisce nel seguente modo un passaggio nella lezione XVII del Corso del sig. Hermite, $2^{2}$ edizione autografata 1883 pag. I 8 .

Siano $\alpha, b$ quantiti reali e siano $F(t, z), G(l, z)$ funzioni olomorfe delle variabili $t, z$, come osserva l'Autore, l'equazione $G(t, z)=0$ fa corrispondere alla serie dei valori reali di $t$ compresi fra $\alpha$ e $\beta$, un numero finito o infinito di porzioni di curve o di curve intere, secondo i casi, le quali sono da riguardarsi quali coupares per la funzione :

$$
\Phi(z)=\int_{x}^{\beta} \frac{F(t, z)}{G(t, z)} d t
$$

Il sig. Hermite per mostrare ciò considera un punto $M$ sopra una di tali curve, al quale rispondono i valori purticolari $t=\theta z=\zeta$, condotta poi la normale in esso punto alla curva prende su questa normale, da una parte e dall'altra della curva, due punti $N, N^{\top}$ infinitamente vicini al punto $M$ ed egualmente distanti da esso per modo che sia $M N=$ - i $M N^{\prime}=\mp \lambda$; trattasi perció di mostrare, che, trovati i valori di $\Phi(z)$ in ciascuno dei punti $N, N^{\prime}$, quando si consideri in essi $\lambda$ infinitamente piccolo la loro differenza es una quantita finita.

Ora dall'equazione della normale in un punto, di affisso $z$, appartenente alla curva ricavasi che lo attisso di un qualunque punto di essa normale $\dot{e}$ :

$$
Z=z+i \lambda \frac{G_{i}^{\prime}(t, z)}{G_{q}^{\prime}(t, z)}
$$

ove intendonsi esclusi $i$ casi nei quali per certi particolari valori di $t$ e di $z$ possa aversi $G_{1}^{\prime}(t, z)=0, G_{i}^{\prime}(t, z)=0$. 
60

Posto :

$$
\frac{G_{1}^{\prime}(t, z)}{G_{z}^{\prime}(t, z)}=p+i q
$$

dopo breve discussione l'Autore trova "che le coordinate del punto di aftisso $Z$ si possono scrivere :

$$
X=x-e_{\lambda} q \quad Y=y+\imath \lambda p
$$

ove $\lambda$ \& positivo ed $\varepsilon$, in valore assoluto eguale all'unith, ha il segno di $p$ se $p \gtrless 0$ e quello di $-q$ se $p=0$. Per avere le coordinate di un punto appartenente alla parte negativa della normale basta supporre $\lambda$ negativo.

Pongasi per brevita :

$$
\begin{array}{ll}
F_{(}^{\prime}(t, z)=P(t, z) & F_{z}^{\prime}(t, z)=Q(t, z) \\
G_{1}^{\prime}(t, z)=R(t, z) & G_{z}^{\prime}(t, z)=S(t, z)
\end{array}
$$

e si scriva semplicemente $P, Q, R, S$ per $P(\theta, \zeta), Q(\theta, \zeta), R(\theta, \zeta)$ $S(\theta, \zeta)$. L'affisso del punto $N$ situato sulla direzione positiva della normale d :

$$
Z=\zeta+i \varepsilon \lambda \frac{R}{S}
$$

onde :

$$
\Psi(N)=\int_{\lambda}^{\beta} \frac{F\left(t, \zeta+i \iota \lambda \frac{R}{S}\right)}{G\left(t, \zeta+i \iota \lambda \frac{R}{S}\right)} d t .
$$


L'espressione analoga relativa al punto $N^{\prime}$ simmetrico di $N$ si ot. tiene mutando $\lambda$ in $-\lambda$, onde si ha :

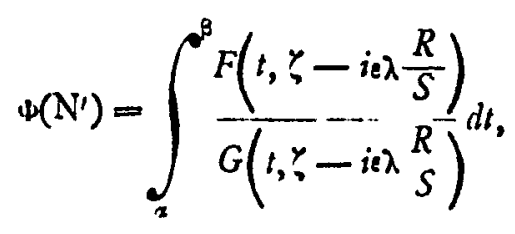

e pero :

$$
=\int_{\alpha}^{\beta}\left\{\frac{F\left(t, \zeta+i \epsilon \lambda \frac{R}{S}\right)}{G\left(t, \zeta+i \varepsilon \lambda \frac{R}{S}\right)}-\left.\frac{F\left(t, \zeta-i i \lambda \frac{R}{S}\right)}{G\left(t, \zeta-i \epsilon \lambda \frac{R}{S}\right)}\right|^{\prime}\right.
$$

dove nell'integrale del $2^{\circ}$ membro bisogna supporre $\lambda$ infinitamente piccolo. Ora esso integrale è la somma dei valori che prende la differenziale

$$
\left\{\frac{F\left(t, \zeta+i \varepsilon \lambda \frac{R}{S}\right)}{G\left(t, \zeta+i \varepsilon \lambda \frac{R}{S}\right)}-\frac{F\left(t, \zeta-i \varepsilon \lambda \frac{R}{S}\right)}{G\left(t, \zeta-i \varepsilon \lambda \frac{R}{S}\right)}\right\} d t
$$

allorchè la variabile cresce per gradi eguali $a d t$ dal limite inferiore $\alpha$ al limite superiore $\beta$, ma gli elementi di siffatta somma relativi ai valori di $t$ che non sono radici dell'equazione $G(t, \zeta)=0$, tendono a zero con $\lambda$, mentre lo stesso non puó dirsi di quegli elementi che rispondono ai valori di $t \mathrm{i}$ quali annullano $G(t, \zeta)$ valori che, dopo le esclusioni fatte sopra, riduconsi al solo $t=\theta$. Onde nell'integrale del $2^{\circ}$ membro della ( 1 ) bisogna supporre $\lambda$ infinitamente piccolo ed è sol- 


\section{2}

tanto da considerare l'elemento, il quale si presenta di forma indeterminata, rispondente al valore $t=\theta$, o altrimenti è permesso sostituire identicamente $t=\theta+(t-\theta)$ in ciascuna delle funzioni

$$
F\left(t, \zeta \pm i \varepsilon \lambda \frac{R}{S}\right), \quad G\left(t, \zeta \pm i \varepsilon \lambda-\frac{R}{S}\right)
$$

nel $2^{\circ}$ membro della (I) e supporre che $\iota-0$ tenda a zero con $\lambda$.

Con tali intendimenti sviluppando le funzioni :

$$
F\left(\theta+(t-\theta), \zeta \pm i \varepsilon \lambda \frac{R}{S}\right), \quad G\left(\theta+(t-\theta), \zeta \pm i \varepsilon \lambda \frac{R}{S}\right)
$$

ed arrestandosi agl'infinitamente piccoli del $\mathrm{I}^{\circ}$ ordine, poichè $G(0, \zeta) \equiv 0$, si trova :

$$
\frac{F\left(t, \zeta \pm i \varepsilon \lambda \frac{R}{S}\right)}{G\left(t, \zeta \pm i \varepsilon \lambda \frac{R}{S}\right)}=\frac{F(\theta, \zeta)}{(t-\theta \pm i \varepsilon \lambda) R}+\frac{(t-\theta) P S \pm i \varepsilon \lambda Q R}{(t-0 \pm i \varepsilon \lambda) R S},
$$

ove i segni si corrispondono. Onde :

$$
\begin{gathered}
\quad \frac{F\left(t, \zeta+i \varepsilon \lambda \frac{R}{S}\right)}{G\left(t, \zeta+i_{\varepsilon} \lambda \frac{R}{S}\right)}-\frac{F\left(t, \zeta-i \varepsilon \lambda \frac{R}{S}\right)}{G\left(t, \zeta-i \varepsilon \lambda \frac{R}{S}\right)} \\
=-\frac{2 i \varepsilon \lambda F(\theta, \zeta)}{\left\{(t--\theta)^{2}+\lambda^{2}\right\} R}-\frac{2 i \varepsilon \lambda(t-\theta)(P S-Q R)}{\left\{(t-\theta)^{2}+\lambda^{2}\right\} R S} .
\end{gathered}
$$

E pero :

$$
\begin{aligned}
W(N)-H\left(N^{\prime}\right)= & -2 i \underline{F}\left(\frac{\theta}{R} \zeta\right) \int_{\alpha}^{\beta} \frac{\lambda d t}{(t-\theta)^{2}+\lambda^{2}} \\
& -2 i_{e}\left(\frac{P}{R}-\frac{Q}{S}\right) \int_{\alpha}^{\beta} \frac{\lambda(t-\theta) d t}{(t-\theta)^{2}+\lambda^{2}} .
\end{aligned}
$$


Trattusi ora di trovare $i$ valori dei due integrali nel $2^{0}$ membro di questa espressione, supposto 0 compreso fra $\alpha, \beta$ e $\lambda$ convergente verso lo zero. Il primo di essi integrali è stato studiato dallo stesso signor Hermite a pag. Iog del detto corso autogrnfito ed egli hat trovato, quando $\theta$ è compreso frra $\alpha, \beta$ :

$$
\lim _{\lambda \rightarrow 0} \int_{x}^{\beta} \frac{\lambda d t}{\left(t-\frac{\lambda}{\theta}\right)^{2}}+\lambda^{2}=\pi
$$

Il secondo integrale, eseguendo l'integrazione, dà :

$$
\int_{x}^{\beta} \frac{\lambda(t-\theta) d t}{(t-\theta)^{2}+\lambda^{2}}=\frac{1}{2} \lambda \log \frac{(\beta-\theta)^{2}+\lambda^{2}}{(\alpha-\theta)^{2}+\lambda^{2}},
$$

onde :

$$
\operatorname{Lim}_{\lambda=0} \int_{x}^{\beta} \frac{\lambda(t-\theta) d t}{(l-\theta)^{2}+\lambda^{2}}=0
$$

e perd :

$$
\omega(N)-\omega\left(N^{\prime}\right)=-\frac{2 i \varepsilon \pi F(\theta, \zeta)}{R(\theta, \zeta)} \cdot(*)
$$

(*) Crr. Hermite Cours professe a la Faculte des Sciences de Paris (Pares 1883, pag. 116), ed anche: Sur quelques points de la thiorie des Fonctions (Acta Societatis Scientiarum Fennicue XII).

Il sig. Gousart in una letterd diretta al sig. Hermite (Acla Mathematica I, pagina 189) dimostra, in modo piü generale, il suddetto teorema applicandovi il teorema di Cauchy; per vero il fattore $\frac{F(\theta, \zeta)}{R(\theta, \zeta)}$ e il coefficiente di $\frac{1}{h}$-nello sviluppo di $\frac{F(\theta+b, \zeta)}{G(+b, \zeta)}$, cioe il residuo della funzione $\frac{F(t, \zeta}{G(t, \zeta)}$ relativo al polo $t=\theta$. 


\title{
SEDUTA DEL 7 FEBBRAIO 1886
}

\author{
PRLSIDLNZA G. ALBLGGIAN!
}

Ammissione di nuovi soci : Prof. Temistocle Zona, Ingegnere Salvatore Rotigliano.

Elezionedel Bibliotecario del Circolo: Dr. Vittorio Martinetti.

E presente alla seduta il Dr. T. A. Hirst di Londra.

G. B. Guccia : Estensione di alcuni teoremi di Hirst sulle trasformazioni quadratiche, alle corrispondenze Cremoniane d'ordine $n$ nel piano.

\section{SEDUTA DEL 2I FEBBRAJO 1886}

\author{
PRESIDENZA G. ALBEGGIANI
}

Ammissione di nuovi soci: Prof. Michele Cantone.

Comunicazioni :

T. A. Hirst. F. R. S. Sur la congruence Roccella, du troisieme ordre et de la troisieme classe.

M. le Dr. Roccella, dans une Thèse intéressante Sugli enti g eometrici dello spazio di rette generali dalle intersezioni de' complessi corrispondenti in due o piib fasci proiettivi di complessi lineari (Piazza Armerina, I 882), a signalé l'existence d'une congruence du $3^{\text {itme }}$ ordre et de la $3^{\text {tkme }}$ classe, dans laquelle je viens de reconnaitre un cas particulier de la congruence Cremonienne que j'ai étudiée dans mon dernier Mémoire On Congruences of the Third Order and Class. (")

(") Proceedings of the London Mathematical Society, Vol. XVI, 1885. 
Pour mettre en évidence cette liaison je rappelle que la congruence Roccella dont il s'agit, peut être definie comme lieu d'une droite qui s'appuie sur trois rayons correspondants de trois faisceaux projectifs $A_{1}(\alpha), B_{2}(\beta), X_{1}(\xi)$ donnís dans l'espace. Parmil les droites de la congruence $(3,3)$ qui forment ce lieu se trouvent les géneratrices de trois cônes du second ordre, et les tangentes de trois coniques. Chacun des trois cônes a pour sommet un des trois centres $A_{2}, B_{1}, X_{2}$ et passe par les deux autres; clacune des trois coniques est située dans un des trois plans $\alpha, \beta, \xi$ et touche les deux autres. Par conséquent, pour établir l' identité de la congruence dont il s'agit avec celle que $j$ ' ai traité dans mon Mémoire, il faut et il suffit de faire voir que la première détermine entre les points de deux quelconques des trois plans, entre $\alpha$ et \& par exemple, une correspondance de Jonquières (isograplraque) du troisième ordre qui possède deux points-unis $C$ et $D$ (voir art. 8 de mon Mémoire).

Pour cela j' observe que le lieu d' une droite d'une congruence $(3,3)$ qui rencontre une droite arbitraire donnée est, en général, une surface gauche du sixième degre, pour laquelle la droite arbitraire est triple. Dans le cas où cette droite est prise arbitrairement sur le plan $\alpha$, la surface gauche se décompose, évidemment, dans les tangentes de la conique située en ce plan et dans une surface gauche, du quatrième degré, pour laquelle la droite directrice $a$ est simple. Mais une géneratrice de cette dernière surface se trouve entièrement dans le plan $\ell$; c'est la tangente, outre que $\overline{\alpha \beta}$, qu' on peut mener at la conique du plan $\beta$ par le point $(a, \bar{\alpha})$. En l'écartant il reste donc', pour section de la surface gauche par le plan $\beta$, une courbe du troisième ordre. C'est la courbe qui correspond projectivement à la droite arbitraire $a$ du plan $\alpha$. Elle possède évidemment en $B_{2}$ un point double, puisque deux des génératrices du cône qui a $B_{2}$ pour sommet rencontrent la droite $a$.

On sait que pour chaque couple de faisce.ux projectifs il arrive, deux fois, que deux rayons correspondants se rencontrent. On voit aussi qu' une droite quelconque qui passe par un tel point de rencontre et s'appuie sur le rayon correspondant à ces deux-là, dans le troisième faisceau, appartient à la congruence; comme y appartient aussi chaque droite du plan des deux rayons incidents qui passe par la trace de ce rayon correspondant. 
66

Or pour les faisceaux $A_{2}(\alpha), B_{1}(\beta)$ les deux points de rencontre des rayons correspondants sout, évidemment, les points-unis $C$ et $D$, de la correspondance entre $\alpha$ et $\beta$, et les traces, sur les plans de ces couples de rayons, des rayon qui correspondent, respectivement, d ces couples, dans le troisieme faisceau, sont les points $C$ et $D^{\prime}$ de mon Mémoire (art. 8).

J'ajoute que, pour les faisceaux $A_{2}(\alpha), X_{2}(\xi)$, les deux points de rencontre des couples de rayons correspondants, sont des points fondamentaux simples $A_{1}^{\prime}, A_{1}^{\prime \prime}$ de la correspondance entre $\alpha$ et $\beta$; de même, pour les faisceaux $B_{2}(\beta), X_{2}(\xi)$ ce sont des points fondamentaux simples $B_{1}^{\prime \prime \prime}, B_{1}^{\prime \prime \prime \prime}$. Les points fondamentaux $B_{1}^{\prime}, B_{1}^{\prime \prime}, A_{1}^{\prime \prime \prime}, A_{1}^{\prime \prime \prime \prime}$, respectivement associés aux précédents, sont toujours les traces, sur le plan de deux rayons incidents, des rayons qui correspondent à ceux-ci dans le troisième faisceau.

Il me reste seulement à faire observer que ce qui caractérise la congruence Roccella, comme congruence $\mathrm{C} r$ emonienne, c'est que

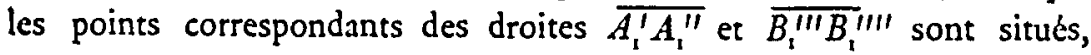
deux $a$ deux, sur les tangentes d'une conique. dont le plan est $\xi$; et ceux des coniques $\left(A_{2} A_{1}^{\prime \prime \prime} A_{1}^{\prime \prime \prime \prime} C D\right)$ et $\left(B_{2} B_{1}^{\prime} B_{1}^{\prime \prime} C D\right)$ sur les génératrices d'un cône du second ordre, dont le sommet est $X_{2}$. Ces relations dans la congruence $(3,3)$, plus générale, que $j$ ' ai étudiée dans mon Memoire, n'ont pas lieu.

G. B. Guccia nggiunge nuovi risultati sull' argomento esposto nella seduta precedente.

\title{
SEDUTA DEL 2I MARZO I886
}

\author{
PRESIDENZA G. ALDEGGIANI
}

Comunicazioni:

G. Maisano comunica alcune ricerche intorno a certe curve covarianti analoghe alle curve Hessiand, Steineriana, e Cayleyana di una curva fondamentale di ordine $n$. 
$\mathrm{Nel}$ sistema doppiamente infinito delle $k^{\mathrm{me}}$ polari dei punti del piano rispetto ad una curva fondamentale $f=a_{x}^{n}=b_{x}^{n}=\ldots=0$ ve ne ha un numero semplicemente infinito che hanno un punto doppio. Il luogo dei relativi poli è una curva dell'ordine $3 k(n-k-1)^{2}$ :

$$
S_{k}(y)=0
$$

che si può ottenere eliminando le $x$ fr.l le 3 equazioni :-

$$
\begin{aligned}
& a_{y}^{k} a_{x}^{n-k-1} a_{1}=0, \\
& a_{y}^{k} a_{x}^{n-k-1} a_{2}=0, \\
& a_{y}^{k} a_{x}^{n-k-1} a_{3}=0 .
\end{aligned}
$$

Il luogo dei punti doppi di queste $k^{\text {me }}$ polari è una curva dell'ordine $3 k^{2}(n-k-1)$ :

$$
\Delta_{k}(x)=0
$$

che si puo ottenere eliminando le $y$ fra le equazioni (2).

L'inviluppo delle rette che uniscono un polo $y$ col punto doppio delln relativa polare es una curva della classe $3 k(n-k-1)(n-1)$ :

$$
C_{k}(11)=0 \text {. }
$$

Chiamando $S_{k}(y)=0, \Delta_{k}(x)=0, C_{k}(u)=0$, rispettivamente, $k^{\text {ma }}$ Steineriana, $k^{\text {ma }}$ Hessiana, $k^{\text {ma }}$ Cayleyana della curva $f=0$, si hanno i seguenti teoremi :

I. Le $(n-k)^{\text {me }}$ polari dei punti della $k^{\mathrm{ma}}$ Hessian a rispetto alla curva fondamentale, toccano la $\mathrm{k}^{\mathrm{ma}}$ Steineriana nei punti corrispondenti.

II. Le $k^{\text {me }}$ polari dei punti della $k^{\text {ma }}$ Steineriana rispetto alla curva fondamentale, tociano (impropriamente) nei punti corrispondenti la Hessiana. 


\section{8}

III. Se la curva fondamentale $f:=0$ ba un punto di ondulazione, nel qual caso si deve annullare un invariante dif, questo punto $k$ comune alle tre curve $f=0, \Delta_{1}=0, \Delta_{2}=0$; reciprocamente, un punto comune a queste tre curve $\dot{e}$ un punto di ondulazione per la curva $f=0$.

G. B. Guccia declina la priorith di una soluzione delle equazioni di condizione per le trasformazioni Cremoniane, contenuta in una nota dell'Ammiraglio de Jonquières ("). Nel mese di ottobre r88; gli era occorso di comunicare verbalmente all'illustre geometra francese i due risultati seguenti :

I. ${ }^{\circ}$ Ove sia $n=\mathbf{2}^{m}$ ( $m$ ntumero intero) si ba sempre la soluzione, conitugata a se stessa,

$$
\alpha_{1}=\alpha_{2}=\alpha_{2^{2}}=\alpha_{23}=\ldots=\alpha_{2^{m-1}}=3\left({ }^{m+*}\right)
$$

$.2^{\circ}$ In ogni trasformazione Cremoniana d'ordine pari, in cui gli ordini di moltiplicita dei punti fondamentali sono tutli dei nutmeri pari, si hanno necessariamente tre punti fondamentali semplici.

In ordine alla prima proposizione gli corre l'obbligo dichiarare aver egli ignorato che di quella soluzione si fossero occupati con precedenza : il Dr. Hirst nella nota Un quadric Transformation (Quarterly Journal, n..$^{\circ}$ 68, 1881) ed il Dr. Sturm nella Memoria Ueber die reciproke und mit ibr zusammenbängende Verwandtschaften (Math. Annalen, XIX, october 1881).

(*) Sur les transformations gionetriques birationnelles d'ordre $n$ (Comples Rendus, t. CI, p. 720,19 octobre 1885 ). Posterionmente il sig. de Jonquières ha continuate le sue importanti ricerche sulle equazioni di $\mathrm{Cremona}$, dando alla luce le note: Modes de solution dune question d'Analyse indeterminie, qui est fondamentule dans la thio. rie des transformations Cremona (Extrait des Comptes 'Rendus, $t$. CI, séances des 2 et 9 novembre 1885 , avec additions), Etule sur une quéstion d' Analyse indeterminde. (Giornale di Battaglini, 1886, vol. XXIV). Cfr. inoltre : Guccia, Sur les transformations geonidriques planes birationnelles. (Comples Rerulus, t. CI, séance du 26 octobre 1885 ).

(**) Posto $m=3$ si ha la soluzione del Cayley (Proceul. Lom.lun Muthematical Society III, p. 143). 


\section{SEDUTA DEL 4 APRILE I 886}

PRESIDENZA F. CALDARERA

Ammissione di nuovi soci: Professore Giuseppe Taschetti.

Comunicazioni :

A. Capelli presenta al Circolo un volume manoscritto del defunto professore Gatano Batd (*) contenente lezioni di Geometria analitica nello spazio e frammenti diversi riguardanti problemi risoluti dall'Autore. Fa notare come le lezioni di Geometria analitica nello spazio possano riguardarsi come la prosecuzione di quelle di Geometria analitica del piano, il cui manoscritto sı trova conservato presso questa Biblioteca Comunale. Venendo quindi a parlare dei sopraddetti frammenti, osserva come specialmente importante il seguente teorema:

L'ellisse di area massima inscrivibile in un triangolo é quella che lo locea nei punti di mezzo.

In proposito a ció lo stesso socio A. Capelli fa delle osservazioni sue proprie indicando un metodo generale che puó servire per l.l dimostrazione di questo e di alcuni altri teoremi analoghi.

F. Giudice $f_{\text {a }}$ conoscere un nuovo metodo stlla determinazione delle radici reali delle equazioni a coefficienti numerici reali, il quale riesce spesso conveniente per la risoluzione approssimata delle equazioni, specialmente quando, per essere queste di grado elevato riescirebbe molto incomodo cosi l'uso dell'equazione alle differenze $\left({ }^{*}\right)$, come l'applicazione del Teorema di Sturm.

(") Gaetano Batd, nato in Palerno l'duno 1783 , fu protessore di m.dematiche sublimi in questa Universith per lo spario di sette ami. Nominuto a tule uficio ne' 1834 cessava di vivere nel dicembre $18+2$. L'aver potuto csamunare it manoscritto presentato al Circolo si deve alla gentulezza della fanjiglia Jacona che ne is proprietura.

(") Lagrange - Otuvres - Tome huitième - Chapitre premser - Probléme 8. 
70

Avendosi un'equazione, che suppongo senza radici multiple, lạ si ponga sotto la forma :

$$
f(x)=\varphi(x)
$$

scelta per modo che $f(x)$ e $\varphi(x)$ siano entrambe costantemente $c_{1}$ escenti mentre $x$ percorre l'intervallo $(a, b)$, dove suppongo $a \geqq 0, b>a$. Perchè cio sia, is necessario e sufficiente che si mantenga

$$
\frac{d f(x)}{d x}>0, \quad \frac{d \varphi(x)}{d x}>0
$$

mentre $x$ percorre il detto intervallo. Si potrd sempre ottenere questo in intiniti modi; e lo si conseguird in ogni caso immediatamente se, dopo d'aver ridotta a zero l'equacione, si trasporteranno tutti $\mathrm{i}$ termini negativi nel secondo membro perche cosi $f(x)$ e $\varphi(x)$ saranno polinomi con soli termini positivi.

$\mathrm{Si}$ formino i valori

$$
f(a) \quad f(b) \quad \varphi(a) \quad \varphi(b)
$$

Essendo arbitrario l'ordine dei due membri della (I), potremo supporre $f(a)<\varphi(a)$.

In causa delle ipotesi (2), se una radice $X$ della (r) cade nell' intervallo $(a, b)$, deve cadere $f(X),=\phi(X)$, tanto nell'intervallo [f(a), $f(b)]$ quanto nell'altro $[\varphi(a), \varphi(b)]$ per cui se questi non avranno niente di comune l'equazione proposta non avrì nessuna radice nell'intervallo $(a, b)$ considerato. Segue che se una radice cade in questo, disponendo per grandezza crescente i valori $f(a), f(b), \varphi(a), \varphi(b), f(X)=\varphi(X)$, avremo o l'uno o l'altro dei seguenti casi :

$$
f(a) \quad \varphi(a) \quad f(X)=\varphi(X) \quad f(b) \quad \varphi(b)
$$

$$
f(a) \quad \varphi(a) \quad f(X)=\varphi(X) \quad \varphi(b) \quad f(b)
$$


onde si riconosce $\left(^{*}\right)$ che la minor radice della (I) superiore ad $a$ è limite di $a_{r}$, al crescere indefinitamente di $r$, purchè sia fatto

$$
f\left(a_{\mathrm{r}}\right)=\varphi(a) \quad f\left(a_{\mathrm{r}}\right)=\varphi\left(a_{\mathrm{r}-\mathrm{l}}\right)
$$

Si riconosce parimenti che la maggior radice della ( 1 ) inferiore $a b$ d linite di $b_{3}$, al crescere indefinitamente di $s$, purche sia fatto
nel caso (I) :
$\varphi\left(b_{1}\right)=f(b)$
$\varphi\left(b_{s}\right)=f\left(b_{s-1}\right)$
e nel caso (II) :
$f\left(b_{1}\right)=\varphi(b)$
$f\left(b_{s}\right)=\varphi\left(b_{s-1}\right)$

Se nessuna radice cadesse nell'intervallo $(a, b)$, il procedimento di calcolo ora indicato ce ne avviserebbe perche le $a_{r}$ diverrebbero maggiori di $b$ e le $b$, diverrebbero minori dii $a$.

Indico con $\alpha$ la minor radice di ( 1 ) superiore ad $a$ ed indico con P la maggior radice inferiore a $b$. Siccome $l a(I)$ non ha radici multiple potremo spingere il calcolo fino ad ottenere $a_{r}$ e $b_{s}$ abbastanza vicine ai loro limiti $a$ e $\&$ perche la minore radice di

$$
\frac{d f(x)}{d x}=\frac{d \varphi(x)}{d x}
$$

superiore ad $a_{r}$ sia purc superiore ad $\alpha$, e la maggior radice, di questa stessa equazione, inferiore a $b$, si.a pure inferiorc a $\beta$. Calcolando in simil guisa i numeri $a_{r, 1}, a_{r, 2}, a_{r, 3}, \ldots$ cost.ntemente crescenti aventi per limite la minor radice della (3) superiore ad $a_{r}$, ed i numeri $b_{s, 1}$, $b_{s, 2}, b_{s, 3}, \ldots$ costantemente decrescenti aventi per limite la maggior radice della (3) inferiore a $b_{s}$, potremo quindi spingere il calcolo fino ad ottenere un numero $A=a_{r, u}$ maggiore di $\alpha$ ed un numero $B=b_{s, 0}$ minore di $\beta$. Pel teorema di Rolle cadra allora la sola radice $\alpha$ fra $a$ ed $A$, o meglio fra $a_{r}$ ed $A$; e cadra la sola $\beta$ fra $b$ e $B$, o meglio fra $b_{s}$ e $B$. Potremo prendere per $A$ e $B$ ll primo numero $a_{r, a}$ ed il primo $b_{s, v}$ per cui si ha :

(") Cauchy - Atulyse Algibrique - Note III - 2une Thiorime. 


$$
\begin{aligned}
& {[f(a)-\varphi(a)] \times\left[f\left(a_{r, u}\right)-\varphi\left(a_{r, u}\right)\right]<0} \\
& {[f(b)-\varphi(b)] \times\left[f\left(b_{s, v}\right)-\varphi\left(b_{s, v}\right)\right]<0}
\end{aligned}
$$

Cosi avremo separate le radici $\alpha$ e $\beta$. Procederemo a limitare le altre radici reali the cadono nell' intervallo $(a, b)$ operando relativamente all' intervallo $(A, B)$ come gid s's operato relativamente d quello.

Volendo limitare tutte le radici positive prenderemo per $a$ e $b$ lo zero ed un numero maggiore dí tutte le radici reali della ( $\mathrm{r}$ ).

A. Cantone comunica i seguenti teoremi sulla cubica gobbat :

I. Se si projetta da un punto qualunque di una retta data $r$ la iubica gobba $\mathrm{r}$ e si cerca della relia $r$ il piano polare rispetto al cono di $3^{\circ}$ ordine cosi generato, questo piano passa costantemeenie per una retta $r$. Le relle $r$ ed $r$ sono li Irasulrsali alle quattro corde situath mi piani tangenli di $\Gamma$, else passano per $r$.

Applicando il principio di duallit.l si ottiene:

Segando con un piano qualsiasi passante per una retta data $r$ la sitperficie $\Sigma$, sviluppabile osiulatrice della cubica gobba $\mathrm{r}$, i poli della retta $r$, rispetto alle curve di $3^{2}$ classe cosi generate, sono sithate su una retta $r_{1}$.

Le relle $r$ ed $r_{1}$ sono le trasversali alle quattro rette direltrici passanti pei punti d'incontro di $r$ con la superficie $\Sigma$.

II. Se da un punto qualunque di una retta $q$ del complesso di primo grado $\Omega$ determinato dalla curva $\Gamma$, si projetta essa carva $\Gamma$, il piano polare di q rispetto al cono di $3^{\circ}$ ordine cosi generato passa costantemente per un'altra relta $q^{\prime}$ del medesimo complesso $n$, e viceversa; inoltre se can un piano qualunque passante per $q$ seghiamo la sviluppabile osculatrice $\mathbf{Z}$, il polo di $q$ rispetto alla curva di $3^{2}$ ilasse, cbe si viene a determinare, giace sulla retta $q^{\prime}$, e viceversa.

In virtù di tali propriet.h alle rette $q$ e $q^{\prime}$ si puó dare il nome di rette polari coniugate rispetto alla cubica gobba $\Gamma$.

III. Una retta $q$ del complesso $\Omega$ sega la superficie $\Sigma$ in due punti reali e due imaginart conugati formanti un rapporto equianarmonico.

Se $\mu$ e $\nu$ sono i due piani tangenti reali di $\Gamma$ nei punti $M$ ed $N$, passanti per la retta $q$ e seganti ancorn $r$ rispettivamente néi punti $M^{\prime}$ 
ed $N$, la retta $q^{\prime}$, polare coniugata di $q$, è l'intersezione del piano tangente di $\Gamma$ in $M^{\prime}$ e segante in $M$, col piano tangente in $N^{\prime}$ e segante in $N$.

Richiamando poi la definizione di punti congiunti e di piani congiunti relativamente ad una cubica gobba $\left(^{*}\right)$, dimostra il teorema :

IV. I piani congiunti a quelli passanti per una retta $q$ del complesso $\Omega$ sono i piani osculatori della cubica gobba luogo dei punti congiunti a quelli situati sulla retta $q$.

Infine richiamando la trasformazione razionale ed involutoria, che si puó stabilire per mezzo di una cubica gobba $\Gamma(*)$, si ha, che le superficie di $3^{\circ}$ ordine $\mathrm{I}_{\alpha}$, corrispondenti ai piani a dello spazio, oltre a passare per la curva $\Gamma$, risultano inscritte alla superficie $\Sigma$, ossia la curva del $6^{\circ}$ ordine, base della trasformazione, si compone di due cubiche gobbe infinitamente vicine.

Se il piano $\alpha$ sega la curva $\Gamma$ in tre punti distinti $A, B$ e $C$, allora la superficie $\|_{x}$ si puo ottenere della trasformazione del piano $\alpha$ per punti congiunti rispetto ad un'altra cubica gobba $\Delta$ passante pei punti $A, B$ e $C$ e quivi tangente alle tre rette, che sono le trasversali alle tangenti di $\Gamma$ nei sudetti punti. Mediante le curve $\Gamma$ e $\Delta$ si puó quindi stabilire una corrispondenza involutoria di punti, tanto sul piano $\alpha$, quanto sulla superficie corrispondente $\Pi_{\alpha}$.

Quando il piano $\alpha$ e osculatore di $\Gamma$, allora esiste un numero semplicemente infinito di curve $\Delta$, le quali possono trasformare il piano $\propto$ nella superficie $\Pi_{x}$.

Supposto il piano $\alpha$ all'infinito enuncia i seguenti teoremi :

V. Se il piano all infinito sega una cubica gobba in tre punti distinti, csiste una superficie di $3^{\circ}$ ordine, la quale bisega le corde non solo della curva data, ma ancora di un'altra cubica gobla, cle ba in comune con la precedente, oltre i tre punti all'infinito, altri due punti e s piani osculatori.

VI. La superficie gobba di $3^{\circ}$ grado, la quale bisega le corde di una

(") Cremona. Memoria sulla cubica gobba. (Nouvelles Ann. de Math. 2.e série t. 1.).

(**) Reye. Lezioni sulla geometria di posizione, p. II, Lez. $14^{*}$. 
parabola gobba, bisegherà ancora le corde di un numero semplicemente infinito di parabole gobbe, ognuna delle quali ì determinata da un punto preso sulla superficie.

\title{
SEDUTA DI:L I8 APRILE I886
}

\author{
PRESIDENZA G. ALBEGGIANI
}

Ammissione di nuovi soci : Professore Damiano Macaluso.

Comunicazioni :

M. Gebbia stabilisce un metodo per formare le equazioni a derivale parziali delle superficie che ammettono una generatrice di forma iostante, fondato sulla consider.zzione che la generatrice, senza deformarsi, vien trasportata nel moto di un sistema invariabile. Questo metodo costituisce quindi un'applicazione della Geometria del movimento.

Siano $\xi, n, \zeta$, le coordinate cartesiane di un punto rispetto a tre assi ortogonali mobili col sistema che trasporta la generatrice ed $x, y$, $z$ le coordinate dello stesso punto rispetto $a$ tre assi ortogonali fissi, a cui si vuol riferire ta superficie. Si definisca la generatrice con tre equazioni

$$
\xi=\varphi(\theta), \quad n=\psi(\theta), \quad \zeta=\%(\theta)
$$

ove $\theta$ è un parametro variabile e sı considerino le note relazioni

$$
\text { (1) } \quad\left\{\begin{array}{l}
\xi=a_{0}+a_{1} x+a_{2} y+a_{3} z=\varphi(\theta)=\psi \\
n-b_{0}+b_{1} x+b_{2} y+b_{3} z=\psi(\theta)=\psi \\
\zeta-c_{0}+c_{1} x+c_{3} y+c_{3} z=\gamma(\theta)=\chi
\end{array}\right.
$$


Per definire il moto della generatrice si suppongano le dodici grandezze $a, b, c$ espresse in funzione di un altro parametro indipendente $\tau$.

Si segnino con $\varphi^{\prime}, \varphi^{\prime \prime}, \ldots \psi^{\prime}, \psi^{\prime \prime}, \ldots$ le derivate di $\varphi, \psi, \chi$ rispetto a $\theta$ e con $a_{0}^{\prime}, b_{0}^{\prime}, c_{0}^{\prime}, a_{1}^{\prime}, \ldots a_{0}^{\prime \prime}, \ldots$ le derivate di $a_{0}, b_{0}, \ldots$ rispetto a $\tau$.

Le grandezze $\theta$, $\tau$ possono sostituirsi alle $x, y$ nella qualit di variabili indipendenti della quistione, ed esse determinano sulla superficie un sistema di coordinnte curviline costituito dalla serie delle diverse posizioni della generatrice e da quella delle traiettorie dei diversi punti di essa.

Differenziando le (I) rispetto a $\theta$ ed a $\tau$, si ottiene

(2)

$$
a_{1} \frac{\partial x}{\partial \theta}+a_{2} \frac{\partial y}{\partial \theta}+a_{3} \frac{\partial z}{\partial \theta}=\varphi^{\prime}
$$$$
b_{1} \frac{\partial x}{\partial \theta}+b_{2} \frac{\partial y}{\partial \theta}+b_{3} \frac{\partial z}{\partial \theta}=\psi^{\prime}
$$$$
c_{1} \frac{\partial x}{\partial \theta}+c_{2} \frac{\partial y}{\partial \theta}+c_{3} \frac{\partial z}{\partial \theta}=\chi^{\prime}
$$

(3) $\left\{\begin{array}{l}a_{1} \frac{\partial x}{\partial \tau}+a_{2} \frac{\partial y}{\partial \tau}+a_{3} \frac{\partial z}{\partial \tau}+a_{0}^{\prime}+a_{1}^{\prime} x+a_{2}^{\prime} y+a_{3}^{\prime} z=0 \\ b_{1} \frac{\partial x}{\partial \tau}+b_{2} \frac{\partial y}{\partial \tau}+b_{3} \frac{\partial z}{\partial \tau}+b_{0}^{\prime}+b_{1}^{\prime} x+b_{2}^{\prime} y+b_{3}^{\prime} z=0 \\ c_{1} \frac{\partial x}{\partial \tau}+c_{2} \frac{\partial y}{\partial \tau}+c_{3} \frac{\partial z}{\partial \tau}+c_{0}^{\prime}+c_{1}^{\prime} x+c_{2}^{\prime} y+c_{3}^{\prime} z=0\end{array}\right.$

Moltiplicando i due membri delle (2) risp. per $a_{1}, b_{1}, c_{1}$, o per $a_{2}, b_{2}, c_{2}$, o per $a_{3}, b_{3}, c_{3}$, e sommando ciascuna volta; poi $f_{2}-$ cendo lo stesso con le (3), si ha per note relazioni: 
(4) $\left\{\begin{array}{l}\frac{\partial x}{\partial \theta}=\Phi, \frac{\partial y}{\partial \theta}=\Psi, \frac{\partial z}{\partial \theta}=\mathrm{X} \\ \frac{\partial x}{\partial \tau}=\Lambda, \frac{\partial y}{\partial \tau}=M, \frac{\partial z}{\partial \tau}=\mathrm{N},\end{array}\right.$

ove

$$
\begin{array}{ll}
\Phi=a_{1} \varphi^{\prime}+b_{1} \psi^{\prime}+c_{1} \gamma^{\prime} & \mathbf{\Lambda}=\rho y-x z-\lambda \\
\Psi=a_{2} \phi^{\prime}+b_{2} \psi^{\prime}+c_{3} \gamma^{\prime} & \mathbf{M}=\pi z-\rho x-\mu \\
\mathrm{X}=a_{3} \phi^{\prime}+b_{3} \psi^{\prime}+c_{3} \gamma^{\prime} & \mathrm{N}=x x-\pi y-\nu \\
\lambda=a_{0}^{\prime} a_{1}+b_{0}^{\prime} b_{1}+c_{0}^{\prime} c_{3} & \pi=a_{3} a_{3}^{\prime}+b_{3} b_{2}^{\prime}+c_{1} c_{2}^{\prime} \\
\mu=a_{0}^{\prime} a_{2}+b_{0}^{\prime} b_{2}+c_{0}^{\prime} c_{2} & x=a_{1} a_{3}^{\prime}+b_{1} b_{3}^{\prime}+c_{1} c_{3}^{\prime} \\
\nu=a_{0}^{\prime} a_{3}+b_{0}^{\prime} b_{3}+c_{0}^{\prime} c_{3} & \rho=a_{2} a_{1}^{\prime}+b_{2} b_{1}^{\prime}+c_{2} c_{1}^{\prime}
\end{array}
$$

Cio posto, il metodo consiste nello stabılire le equazioni, che servono al cambiamento delle variabili indipendenti $x, y$ nelle altre $\theta, \tau$. Si ha dapprima

$$
\begin{aligned}
& \frac{\partial z}{\partial \theta}=\frac{\partial z}{\partial x} \frac{\partial x}{\partial \theta}+\frac{\partial z}{\partial y} \frac{\partial y}{\partial \theta}, \\
& \frac{\partial z}{\partial \tau}=\frac{\partial z}{\partial x} \frac{\partial x}{\partial \tau}+\frac{\partial z}{\partial y} \frac{\partial y}{\partial \tau},
\end{aligned}
$$

le quali, scrivendo come di solito $p, q$ invece di $\frac{\partial z}{\partial x}, \frac{\partial z}{\partial y}$ e per le relazioni (4) diventano 
(s)

$$
\left\{\begin{array}{l}
\Phi p+\Psi q-\mathbf{x}=0 \\
\Lambda p+\mathbf{M} q-\mathbf{N}=0
\end{array}\right.
$$

che sono due equazioni a derivate parziali del $I^{0}$ ordine racchiudenti le funzioni generalmente arbitrarie $\Phi, \Psi, X, \Lambda, M, N$.

Il significato geometrico di queste equazioni si rende manifesto osservando che $\Phi, \Psi, X$ sono proporzionali ai coseni degli angoli che la tangente alla curva forma con gli assi fissi; che $-\Lambda,-M,-N$ esprimono le componenti della velocitł del punto generatore secondo gli assi fissi, e sono quindi proporzionali ai coseni degli angoli che la tangente alla traiettoria di questo punto forma coi detti assi; che infine $p, q,-\mathrm{I}$ sono proporzionali ai coseni degli angoli determinati con gli assi fissi dallin normale alla superficie. Le equazioni (5) esprimono dunque che la normale alla superficie é perpendicolare : $I^{0}$ alla tangente alla curva generatrice; $2^{\circ}$ alla tangente alla traiettoria del punto generatore. Queste proprietì, stabilite a priori, com'è agevole, potrebbero condurre a quelle equazioni direttamente.

$\mathrm{Da}$ esse si possono dedurre tre altre equazioni a derivate parziali del $2^{\circ}$ ordine che racchiuderanno insieme alle precedenti altre funzioni arbitrarie, e che suranno quelle esprimenti la trasformazione delle derivate seconde di $z$. Per ottenerle basta differenziare le $(5)$ rispetto a $\theta$ ed a $\tau$. In questa maniera si perviene apparentenzente a quattro equazioni, che peró si riducono effettivamente $a$ tre in virtù delle identit..

$$
\frac{\partial}{\partial \tau} \frac{\partial x}{\partial \theta}=\frac{\partial}{\partial \theta} \frac{\partial x}{\partial \tau}, \text { etc., }
$$

le quali si traducono nelle relazioni

$$
\text { (6) }\left\{\begin{array}{l}
\frac{\partial \Phi}{\partial \tau}=\frac{\partial \Lambda}{\partial \theta}=\Psi_{p}-\mathrm{Xx}, \\
\frac{\partial \Psi}{\partial \tau}=\frac{\partial M}{\partial \theta}=\mathrm{X} \pi-\Phi p \\
\frac{\partial \mathrm{X}}{\partial \tau}=\frac{\partial \mathrm{N}}{\partial \theta}=\Phi x-\Psi \pi .
\end{array}\right.
$$


Le tre eguaglianze che in tal modo si ottengono sono le seguenti:

(7)

$$
\mid \begin{aligned}
& \Phi^{\prime} p+\Psi^{\prime} q-\mathbf{X}^{\prime}+\Phi^{2} r+2 \Phi \Psi s+\Psi^{2} t=0 \\
& \Lambda \Phi r+(\Lambda \Psi+M \Phi) s+M \Psi l+\left|\begin{array}{lll}
\Phi, & \Psi, & \mathbf{X} \\
\pi, & x, & p \\
p, & q,-\mathrm{I}
\end{array}\right|=0 \\
& \Lambda^{\prime} p+\mathbf{M}^{\prime} q-\mathrm{N}^{\prime}+\left[\pi p q-x\left(\mathrm{I}+p^{2}\right)-p q\right] \Lambda \\
& +\left[\pi\left(\mathrm{I}+q^{2}\right)-x p q+p p\right] \mathrm{M}+\Lambda^{2} r+2 \Lambda M s+M^{2} t=0,
\end{aligned}
$$

ove $\Phi^{\prime}, \Psi^{\prime}, \mathbf{X}^{\prime}$ indicano le derivate parziali di $\Phi, \Psi, \mathbf{X}$ rispetto a $\theta$, $\Lambda^{\prime}, \mathbf{M}^{\prime} \mathbf{N}^{\prime}$ le derivate parzinli di $\Lambda, M, N$ rispetto $\mathbf{\tau}$ : son queste le nuove funzioni arbitrarie. Poi $r, s, t$ rappresentano, come di solito, le derivate seconde di $z$ rispetto ad $x$ ed $y$.

Cosi potranno formarsi successivamente quattro equazioni del terzo ordine, cinque del quarto, etc., che conterranno come funzioni generalmente arbitrarie le derivate parziali successive di $\Phi, \Psi, X$ rispetto a 0 e quelle di $\Lambda, M, N, \pi, x, \rho$ rispetto a $\tau$.

Definita la natura della superficie, imponendo speciali condizioni alla generatrice, o al moto di essa o ad entrambe le cose insieme, per ottenerne l'equazione a derivate parziali si elimineranno le arbitrarie fra tante equazioni dei gruppi (5), (7) e seguenti, quinte ne son necessarie, tenuto conto delle condizioni sudette.

Applica brevemente il processo esposto alla ricerca delle equazioni a derivate purziali delle superficie cilindriche, coniche, di rivoluzione, etc.

Superficie cilindriche. Queste ammettono due serie di generatrici 
di forma costante. Una è la retilinea, epperó $\Phi, \Psi \mathrm{X}$ sono costanti e la prima delle ( 5 ) è gi.l l'equazione cercata. L' altra è una curva qualunque moventesi in traslazione, onde si ha $\pi=x=p=0$, mentre $\lambda, \mu, \nu$ sono costanti, e la seconda delle ( 5 ) diventa

$$
\lambda p+\mu q-\nu=0
$$

cioè sotto altro riguardo si perviene allo stesso tipo d'equazione.

Superficie coniche. La generatrice è rettilinea e passa per un punto fisso $(a, b, c)$, onde le sue equazioni nel sistema fisso sono:

$$
\frac{x-a}{\Phi}=\frac{y-b}{\Psi}=\frac{z-c}{\mathrm{X}}
$$

e la prima delle (s) si puó serivere

$$
(x-a) p+(y-b) q=z-c,
$$

ch'e l'equazione cercata.

Superficie rigale generali. Le $\Phi, \Psi, \mathrm{X}$ sono indipendenti da $\theta$, onde $\Phi^{\prime}=\Psi^{\prime}=X^{\prime}=0$ e la prima delle (7) si riduce a

$$
\Phi^{2} r+2 \Phi \Psi s+\Psi^{2} t=0 \text {. }
$$

Differenziandola rispetto a 0 , si ottiene

$$
\Phi^{3} u+3 \Phi^{2} \Psi v+3 \Phi \Psi^{2} w+\Psi^{3} u=0,
$$

ove $u, v, w, u$ indicano, come di solito, le derivate terze di $z$. Eliminando $\Phi, \Psi$ fra queste due forme binarie, si perviene all'equazione nota delle superficie rigate.

Superficie sviluppabili. Come per le rigate generali la prima delle (7) si riduce a

$$
\Phi^{2} r+2 \Phi \Psi s+\Psi^{2} t=0,
$$


80

che si pud scrivere

(a)

$$
(\Phi r+\Psi s) \Phi+(\Phi s+\Psi t) \Psi=0 .
$$

Inoltre, affinchè le generatrici consecutive s'incontrino, è necessario che il moto istantaneo della generatrice si riduca ad una rotazione semplice e che l'asse istantaneo di questa incontri la generatrice. La prima condizione richiede che sia

$$
\lambda \pi+\mu x+\nu p=0,
$$

che, aggiungendovi un'identith, si può scrivere

(b)

$$
\Lambda \pi+M x+N p=0 .
$$

Per esprimere la seconda condizione si osservi che le coordinate (di retta) della generatrice sono

$\Phi, \quad \Psi, \quad \mathrm{x}, \quad y \mathrm{x}-z \Psi, \quad z \Phi-x \mathrm{x}, \quad x \Psi-y \Phi$

e quelle dell'asse istantaneo

$$
\pi, \quad x, \rho, \lambda, \mu, \quad \nu,
$$

onde la condizione d'incontro prende la forma

(c)

$$
\Lambda \Phi+M \Psi+N X=0 .
$$

Eliminando $\Lambda, \mathbf{M}, \mathbf{N}$ fra le $(b),(c)$ e la seconda delle ( 5 ), si ottiene

$$
\left|\begin{array}{ccc}
\Phi, & \Psi, & \mathrm{X} \\
\pi, & x, & \rho \\
p, & q, & -\mathrm{I}
\end{array}\right|=0
$$


e la seconda delle (7) si potrd scrivere

(d)

$$
(\Lambda r+M s) \Phi+(\Lambda s+M t) \Psi=0 .
$$

Ora dalle $(a),(d)$ si ottiene

$$
\left|\begin{array}{cc}
\Phi r+\Psi s, & \Phi s+\Psi t, \\
\Lambda r+\mathrm{M} s, & \Lambda s+\mathrm{M} t,
\end{array}\right|-\left|\begin{array}{cc}
\Phi, \Psi \\
\Lambda, \mathrm{M}
\end{array}\right| \cdot\left|\begin{array}{cc}
r, & s \\
s, & t
\end{array}\right|=0 .
$$

Il primo determinante di questo prodotto non puó essere genernlmente nullo, onde resta

$$
r t-s^{2}=0,
$$

ch'e la nota equazione delle sviluppabili.

Superficie di rivoluzione. L'equazione si ricava dalla seconda delle (5). Ammettendo che l'asse della superficie passi per l'origine degli assi mobili e che questa concida con l'-rigine dei fissi, si ha $\lambda=\mu$ $\Rightarrow v=0$ e la cennata equazione si riduce $a$

$$
(\rho y-x z) p+(\pi z-\rho x) q-x x-\pi y,
$$

ove $\pi, x, \rho$ si debbono riguardare come costanti.

Superficie canali. L'equazione a derivate parziali del prim'ordine di queste superficie, che si ricaval di solito riguardandole come inviluppi di sfere, non si puó dedurre col processo qui svolto, se non implicando le primitive (I), dapoiche in questo caso l'eliminazione delle $a, b, c$, fra queste equazioni diventa possibile, e se ne ricava una primitiva, della quale le $(5)$ sono le derivate, e che diventa utile per l' eliminazione delle arbitrarie. Cosi avviene che nell'equazione finale, oltre alle derivate parziali di $z$ entri la $z$ stessa.

La generatrice è un circolo di raggio $R$, che supponghiamo tracciato nel piano $\zeta_{n}$ col centro all'origine degli assi mobili, onde : 
82

$$
\begin{array}{ll}
\varphi=R \cos \theta, \quad \psi=R \operatorname{sen} \theta, & \chi=0 \\
\varphi^{\prime}=-R \operatorname{sen} \theta, & \psi^{\prime}=R \cos \theta, \quad \chi^{\prime}=0,
\end{array}
$$

per cui

(e) $\quad \Phi^{2}+\Psi^{2}+\mathrm{X}^{2}=\varphi^{\prime 2}+\psi^{\prime 2}+\chi^{\prime 2}=R^{2}$.

L'origine degli assi mobili rimanga sempre nel piano $x y$, al quale il piano $\xi_{n}$ resti sempre perpendicolare. Si ha quindi

$$
c_{3}=0 ; \quad a_{0} a_{3}+b_{0} b_{3}+c_{0} c_{3}=0
$$

Il movimento istantaneo è una rotazione, il cui asse è sempre parallelo all'asse $z$, onde

$$
v=\pi=x=0, \quad N=0
$$

e si ha

(f) $\quad \Lambda \Phi+M \Psi=0$.

La seconda delle $(5)$ diventa

(g)

$$
\Lambda p+\mathrm{M} q=0
$$

e dalle $(f),(g)$ si ricava

$$
\boldsymbol{t} q-\Psi p=0
$$

Da questa e dalla prima delle (s) si ottiene

$$
\Phi=\frac{x p}{p^{2}+q^{2}}, \quad \Psi=\frac{x q}{p^{2}+q^{23}}
$$


Sostituendo questi valori nella (e), risulta

(b)

$$
\left(\mathrm{I}+p^{2}+q^{2}\right) \mathrm{X}^{2}=R^{2}\left(p^{2}+q^{2}\right) \text {. }
$$

Ora la $\mathrm{X}$ si puo esprimere in $z$ per mezzo delle ( $\mathrm{r}$ ). Infatti da queste si ricava

$$
z=a_{3}\left(\varphi-a_{0}\right)+b_{3}\left(\psi-b_{0}\right)+c_{3}\left(\chi-c_{0}\right)
$$

ovvero, per le precedenti eguaglianze,

$$
z=a_{3} \phi+b_{3} \psi
$$

ed inoltre si ha

$$
\mathrm{X}=a_{3} \phi^{\prime}+b_{3} \psi^{\prime}=-a_{3} \psi+b_{3} \varphi .
$$

Da queste si deduce, per note relazioni,

$$
\mathrm{x}^{2}+z^{2}=R^{2}
$$

e la (b) diventa

$$
\left(1+p^{2}+q^{2}\right) z^{2}=R^{2},
$$

ch'è l'equazione nota delle superficie canali.

Termina deducendo l'ordine differenziale dell'equazione a derivate parziali generale delle superficie che ammettono una generatrice di forma costante, dapoichè difficoltd insormontabili di eliminazione vietano di poterla formare.

Per brevith di linguaggio si indichino coi simboli $\theta, \tau$ le equazioni $(5)$, coi simboli $\theta^{2}, \theta \tau, \tau^{2}$ le equazioni (7), e cosi di seguito analogamente. Cosi queste equazioni e quelle che si potranno ottenere proseguendo 
84

il medesimo processo saranno indicate da simboli, coi quali si puo formare il seguente quadro :

$$
\begin{gathered}
\theta, \quad \tau, \\
\theta^{2}, \theta \tau, \quad \tau^{2}, \\
\theta^{3}, \quad \theta^{2} \tau, \quad \theta \tau^{3}, \quad \tau^{3}, \\
\theta^{4}, \quad \theta^{3} \tau, \quad \theta^{2} \tau^{2}, \quad \theta \tau^{3}, \quad \tau^{4},
\end{gathered}
$$

Se ben si considera il modo come queste equazioni vengono successivamente formate, si riconoscerd che l'equazione indicata dal simbolo $\theta^{m} \tau^{n}$ conterrd le arbitrarie $\Phi, \Psi, X$ e tutte le loro derivate rispetto a $\theta$ fino all' ordine $m$, e dippiù le arbitrarie $\Lambda, M, N, \pi, x, p$ e tutte le loro derivate rispetto a $\tau$ fino all'ordine $n$, sicche questa equazione conterrd in tutto $3 m+6 n$ arbitrarie.

Intanto e facile verificare che il numero delle equazioni cresce più rapidamente del numero delle arbitrarie, e quindi sard infine possibile di eliminare quest' ultime, ottenendo in tal guisa equazioni a derivate parziali, che rappresenteranno la condizione, intesa nel senso più largo, che una superficie ammetta una generatrice di forma costante. Evidentemente sard possibile in più maniere di separare dalle precedenti un gruppo di tali equazioni, che permettano di elininare tutte le arbitrarie che vi son contenute. Ma allora si presenta la quistione di cercare fra siffatti gruppi quello, o quelli, per cui l'ordine differenziale dell'equazione risultante sia il minimo possibile.

Per risolvere questa quistione osserviamo primieramente che, per separare dal precedente schema gruppi di equazioni che contengano certe arbitrarie, e queste sole, è necessario che coteste arbitrarie non siano scelte comunque, $\mathrm{ma}$ che sinno le $\Phi, \Psi, \mathrm{X}$ e tutte le loro derivate rispetto a $\theta$ fino ad un cert'ordine $m$ e le $\Lambda, M, N, \pi, x, \rho$ e tutte le loro derivate rispetto $a \tau$ fino ad un cert'altro ordine $n$, poichè precisamente in questo modo le arbitrarie compariscono nelle equa- 
zioni. Ed allora le equazioni contenenti queste sole arbitrarie sono tutte quelle rappresentate dai simboli $\theta^{\mu} \tau^{\nu}$, ove $\mu-I, 2, \ldots m ; v-1$, $2, \ldots n$. I simboli di queste equazioni formano nel quadro un parallelogrammo, che ha un vertice nel simbolo $\theta^{m} \tau^{*}$ ed il vertice opposto smussato, ed il loro numero è

$$
(m+1)(n+1)-1=m n+m+n .
$$

Per brevith di espressione chiamerb i gruppi di equazioni cosi fatti gruppi completi.

Ora cerchiamo fra i gruppi completi quello, nel quale il numero 'delle equazioni superi del meno possibile quello delle arbitrarie, mentre l'ordine differenziale sia il ininimo possibile. Sia $\theta^{m} \tau^{\prime \prime}$ il simbolo d'ordine più elevato del gruppo, onde l'ordine differenzinle sard

$$
n+n=k
$$

Dovrà esser sodisfatta la diseguaglianza

$$
m n+m+n>3 m+6 n \text {, }
$$

la quale, sostituendo $m$ con $k-n$, ci dasd

$$
k>n \cdot \frac{n+3}{n-2}
$$

Dippiù, perche sia $m$ positivo, è necessario che si abbia

$$
k>n \text {. }
$$

Abbiamo dunque per $k$ due limiti inferiori, dei quali basta tenere il più grande, ch'è il primo dei precedenti.

Il minimo valore positivo dell' espressione $n \cdot \frac{n+3}{n-2}$ si ha per 
86

$n-2+V \overline{10}$, ed aggiungendo la condizione di $n$ intero, abbiamo $n-6$, onde il minimo sudetto sarebbe $6 \cdot \frac{9}{4}$, ed aggiungendo la condizione che questo minimo sia pure intero, ottenghiamo 14. Ora possiamo prendere $k$ uguale al suo limite inferiore $\mathrm{I}_{4}$, salvo a verificare il risultato, ed abbiamo

$$
k=\mathrm{r}_{4}, \quad n=6, \quad m=8 .
$$

Con queste cifre il numero delle equazioni sard 62 e quello delle arbitrarie 60 , onde il valore preso per $k$ risponde alle condizioni richieste. Questo gruppo completo contiene dunque un'equazione di più di quante sono strettamente necessarie, e possiamo omettere quella di ordine piủ elevato, ch'ê rappresentata dal simbolo $0^{8} \tau^{6}$. Il gruppo rimanente condurrà ad una equazione del $13^{\circ}$ ordine.

Or è eviderite che nessun altro gruppo potrì dar luogo ad una risultante d'ordine minore di questo, poichè, se un tal gruppo esistesse, dopo l'analisi precedente questo non potrebbe esser completo; $\mathrm{ma}$ se fosse incompleto, il gruppo completo contenente le stesse arbitrarie ne permetterebbe a fortiori l'eliminazione, e quindi o questo coinciderebbe con quello dianzi ottenuto, o non risponderebbe alla condizione di minimo.

Cosi resta affermato che

La condizione generale clse una superficie ammetia una generatrice di forma costante \& rappresentabile con un'equazione a derivate parziali del $\mathrm{I} 3^{\circ}$ ordine.

G. Maisano : Sulle tangenti doppie e d'inflessione della curva generale del $S^{\circ}$ ordine.

Indicando con $A, B, C$ i tre invarianti fondamentali della quintica binaria, le condizioni necessarie e sufficienti perchè questa ammetta due elementi doppi vengono espresse dalle due condizioni:

$$
A^{2}-64 B=0, \quad 2^{8} \cdot 3^{2} C+A^{3}=0 .\left(^{*}\right)
$$

(") Cfr. G. Maisano: Sulla forma binaria di quint'orline. (Memorie della Reale Mecadema der Lincei, XIV). 
Per mezzo dell' Uebertragungsprincip dagl'invarianti $A, B, C$ si ottengono $\mathrm{i}$ tre contravarianti della quintica termaria

$$
\begin{aligned}
& \Phi=\left(\Theta^{\prime} u\right)^{2} u_{0}^{4} u_{\theta^{\prime}}^{4}, \\
& \Psi=\left(\Pi \Pi^{\prime} u\right)^{2}\left(\Pi^{\prime \prime} \Pi^{\prime \prime \prime} u\right)^{2}\left(\Pi \Pi^{\prime \prime} u\right)\left(\Pi^{\prime} \Pi^{\prime \prime \prime} u\right) u_{\pi}^{6} u_{\pi^{\prime}}^{6} u_{\pi^{\prime \prime}}^{6} u_{\pi^{\prime \prime \prime}}^{6}, \\
& \chi=\left(\Pi \Pi^{\prime} u\right)^{2}(\Pi \Theta u)\left(n^{\prime} \Theta u\right) u_{s}^{3} u_{\pi^{\prime}}^{6} u_{0}^{4},
\end{aligned}
$$

ponendo simbolicamente

$$
f=a_{x}^{5}, \quad(a b u)^{4} a_{x} b_{x}=\Theta_{x}^{2} u_{0}^{4}, \quad(a b u)^{2}(a c u)^{2}(b c u)^{2} a_{x} b_{x} c_{x}=n_{x}^{3} u_{\pi}^{6} .
$$

Le condizioni ( $\mathrm{l}$ ) si mutano dunque nelle

$$
\Phi^{2}-64 \%=0, \quad \Phi^{3}+2^{8} \cdot 3^{2} \Psi=0
$$

rappresentanti due curve rispettivamente della $20^{\circ}$ e della $30^{a}$ classe, la prima delle quali è l'equazione della curva fondamentale $f=0$ in coordinate di retta.

Dai risultati ottenuti nella citata Memoria si conchiudono facilmente $\mathrm{i}$ seguenti :

I. Le tangenli d'inflessione della curva generale del $5^{\circ}$ ordine $f=0$ sono tutte le tangenti comuni alle tre curve

$$
\phi=0, \quad \Psi=0, \quad \chi=0,
$$

rispettivamente della classe $10^{\mathrm{a}}, 20^{\mathrm{a}}, 30^{\mathrm{a}}$.

II. Le tangenti doppie della curva generale del $5^{\circ}$ ordine $f=0$ sono 
88

comuni alle due curve (4), le quali sono ancbe loceate dalle langenti di inflessione della stessa curva $f=0$.

III. Tutte le tangenti comuni alle curve (4) sono soltanto quelle ora menzionate e propriamente le tangenti doppie contate due volte e le tangenti d'inflessione contate otto volte. 